\title{
Physical determining factors of the arterial pulse waveform: theoretical analysis and calculation using the 1-D formulation
}

\author{
Jordi Alastruey • Tiziano Passerini • \\ Luca Formaggia · Joaquim Peiró
}

Received: 5 August 2011 / Accepted: 26 April 2012 / Published online: 25 September 2012

(C) The Author(s) 2012. This article is published with open access at Springerlink.com

\begin{abstract}
The shape of the arterial pulse waveform is intimately related to the physical properties of the cardiovascular system. It is clinically relevant to measure those properties that are related to cardiovascular function, such as the local elasticity and viscosity of the arterial wall, total compliance and net peripheral resistance of the systemic arterial tree. Most of these properties cannot be directly measured in vivo, but they can be calculated from pressure, flow and wall displacement measurements that can be obtained in vivo. We carry out a linear analysis of the one-dimensional (1-D) equations of blood flow in Voigt-type visco-elastic vessels to study the effects on pulse wave propagation of blood viscosity, flow inertia, wall visco-elasticity, total arterial compliance, net resistance, peripheral outflow pressure, and flow rate at the aortic root. Based on our analysis, we derive methods to calculate the local elastic and viscous moduli of the arterial wall, and the total arterial compliance, net resistance, time constant and peripheral outflow pressure of the systemic arterial tree from pressure, flow and wall displacement data that can be measured in vivo. Analysis of in vivo data is beyond the scope of this study, and therefore, we verify the results of our linear analysis and assess the accuracy of our estimation methods using pulse waveforms simulated in a nonlinear visco-elastic 1-D model of the larger conduit arteries of the upper body, which includes the circle of Willis in the cerebral circulation.
\end{abstract}

\author{
J. Alastruey $(\varangle)$ \\ King's Health Partners, St. Thomas' Hospital, London SE1 7EH, UK \\ e-mail: jordi.alastruey-arimon@kcl.ac.uk \\ T. Passerini · L. Formaggia \\ Dipartimento di Matematica F. Brioschi, Politecnico di Milano, Milan, Italy \\ L. Formaggia \\ e-mail: luca.formaggia@polimi.it \\ T. Passerini \\ Department of Mathematics and Computer Science, Emory University, Atlanta GA, USA \\ e-mail: tiziano@mathcs.emory.edu \\ J. Peiró \\ Department of Aeronautics, Imperial College, London SW7 2AZ, UK \\ e-mail: j.peiro@imperial.ac.uk
}

Department of Biomedical Engineering, Division of Imaging Sciences and Biomedical Engineering, King's College London, 
Keywords Circle of Willis · Flow inertia - Nonlinear one-dimensional modelling · Peripheral outflow pressure · Pulse wave propagation $\cdot$ Systemic arteries $\cdot$ Voigt-type visco-elasticity $\cdot$ Wall compliance $\cdot$ Windkessel pressure

\section{Introduction}

The propagation of the pulse wave in systemic arteries induces changes in the luminal area, blood pressure and volume flow rate in time and along the axial direction of arteries. The features of these space-time variations are intimately related to the physical properties of the cardiovascular system, such as blood density and viscosity, cardiac output, arterial wall visco-elasticity, and peripheral impedances and outflow pressures.

Measurement of these properties can be valuable to assess cardiovascular function. Indeed, the pulse wave speed (which is directly related to local arterial stiffness and compliance) has been identified as an important predictor of life-threatening cardiovascular events [1-3]. Changes in the total systemic arterial compliance (which is related to the total stiffness of the systemic arterial tree) have been linked to pathological conditions, such as hypertension $[4,5]$. Vessels with a dysfunctional capacity to dampen the high frequencies of the pulse waveform (which is directly related to the viscosity of the vessel wall) are more likely to suffer from vascular disease such as atherosclerosis [6].

Most of these properties cannot be directly measured in vivo, but they can be calculated from pressure, flow and wall displacement measurements that can be obtained in vivo. These calculations require a good understanding of the individual role of mechanical properties on the pulse waveform. However, this understanding can be extremely challenging to achieve in vivo, since some vessels are inaccessible to clinical measurements and several properties of interest are not directly measurable, can be dangerous to manipulate and can elicit reflex compensation. Onedimensional (1-D) blood flow models can effectively simulate the pulse wave propagation phenomenon in larger systemic arteries [7-9] and, hence, offer an effective approach to study the individual role of clinically relevant properties, derive methods to calculate them and assess the error of the calculations.

Several optimisation algorithms have been proposed to estimate clinically relevant properties from in vivo or in vitro data, e.g. Kalman filtering techniques for the peripheral boundary conditions [10], an adjoint state approach for the local compliance [11] and local sensitivity indices for several mechanical properties of the arteries of the arm [12]. Other works have proposed estimation algorithms that are based upon a mechanical understanding of the effect that the parameter to be calculated has on haemodynamic quantities that can be measured clinically. For example, this is the approach followed in $[13,14]$ to calculate, using the 1-D formulation, the local pulse wave speed from simultaneous pressure and velocity measurements.

In this work we follow the latter approach to develop a methodology to calculate, from haemodynamic data that can be measured in vivo, properties of the systemic arterial tree that are important for assessing cardiovascular function and running patient-specific simulations. We first carry out a linear analysis of the 1-D equations of blood flow in visco-elastic vessels to study the effects on pulse wave propagation of blood viscosity, cardiac output, flow inertia, wall visco-elasticity, total arterial compliance, net peripheral resistance, and outflow pressure (Sect. 2). Based on this analysis, we describe our methods appropriate for calculating the local compliance, inviscid pulse wave speed and elastic and viscous moduli of the arterial wall from pressure and area waveforms, and the total compliance from the flow at the aortic root and a pressure waveform (Sect. 3).

In Sect. 4 we verify the results of our linear analysis using pulse waveforms simulated in the nonlinear model of the larger conduit arteries of the upper body described in [15], which includes the circle of Willis in the cerebral circulation (Fig. 1). Moreover, we assess the parameter estimation methods in the upper body model, by comparing estimates with theoretical values that are directly calculated from the parameters of the model.

\section{1-D formulation}

The nonlinear governing equations of the 1-D incompressible flow in a compliant and impermeable vessel are [7] 


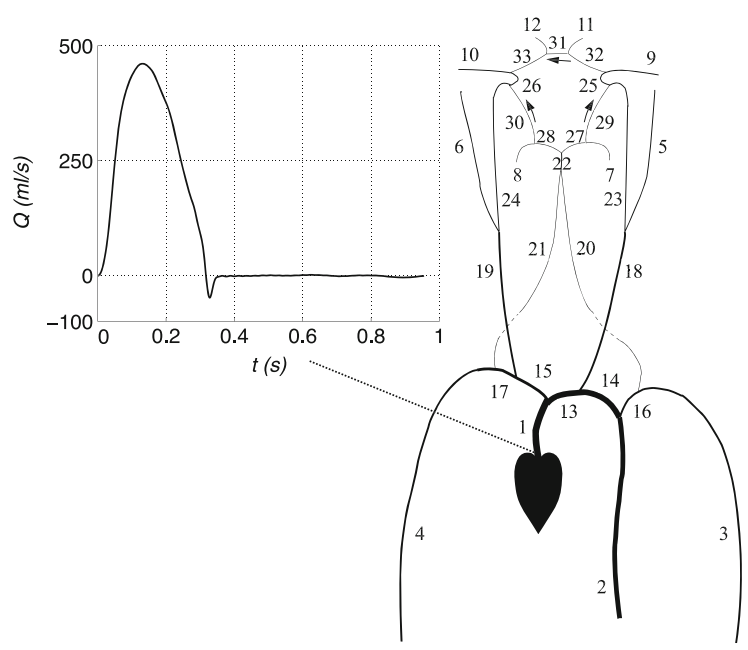

Fig. 1 Schematic representation of the 33-artery network used to simulate pulse wave propagation in the cerebral circulation. The name and properties of each artery are given in Tables 1 and 1 in [15], and are based on data for the normal human; the wall viscous moduli from [16,17] and the rest of properties from [15]. The volume flow rate shown in the plot (whose time average is 5.61/min) is the ensemble average of in vivo measurements at the ascending aorta of a healthy human. It is enforced as a periodic inflow boundary condition (modified from [15])

$$
\left\{\begin{array}{l}
\frac{\partial A}{\partial t}+\frac{\partial Q}{\partial x}=0 \\
\frac{\partial Q}{\partial t}+\frac{\partial}{\partial x}\left(\alpha \frac{Q^{2}}{A}\right)+\frac{A}{\rho} \frac{\partial P}{\partial x}=\frac{f}{\rho},
\end{array}\right.
$$

where $x$ is the axial coordinate along the vessel, $t$ is time, $A(x, t)$ is the cross-sectional area of the lumen, $Q(x, t)$ is the mass flux, $P(x, t)$ is the average internal pressure over the cross section, $\alpha$ is a non-dimensional correction coefficient that accounts for the fact that the velocity profile is not flat, and $\rho$ is the constant mass density of blood. The friction force per unit length, $f(x, t)$, is given by [18]

$$
f=2 \mu \pi \mathcal{R}\left[\frac{\partial u}{\partial r}\right]_{r=\mathcal{R}}
$$

where $\mu$ is the constant blood viscosity, $\mathcal{R}(x, t)$ is the luminal radius, and $u(x, r, t)$ is the axial velocity $(r$ is the radial coordinate). A typical profile for axisymmetric flow satisfying the no-slip condition is

$u=U \frac{\zeta+2}{\zeta}\left[1-\left(\frac{r}{\mathcal{R}}\right)^{\zeta}\right]$

where $U(x, t)=Q / A$ is the average axial velocity and $\zeta$ is a constant. Substitution of Eq. (3) into (2) yields $f=-2(\zeta+2) \mu \pi U$. Following [18], $\zeta=9$ provides a good compromise fit to experimental findings. Notice that $\zeta=2$ corresponds to a parabolic profile, which leads to Poiseuille flow resistance $f=-8 \mu \pi U$.

Following [19], we set $\alpha=1$ in the convective inertia term of Eq. (1). This is a common approximation that leads to considerable mathematical simplifications, especially with the treatment of boundary conditions. This approximation will not affect our linear analysis, since the convective acceleration term is nonlinear.

System (1) is typically closed by defining an explicit algebraic relationship between $P$ and $A$. Here we use $P=\mathcal{F}\left(A ; \partial_{t} A, x\right)$, where the function $\mathcal{F}$ depends on the model used to simulate the dynamics of the arterial wall. We have included a direct dependence on $x$, since some of the mechanical properties of the wall may vary with $x$. More precisely, we resort to the Voigt-type visco-elastic tube law [9]

$$
P=P_{\mathrm{e}}(A ; x)+\frac{\Gamma(x)}{A_{0}(x) \sqrt{A}} \frac{\partial A}{\partial t},
$$


Table 1 Estimates of the theoretical local inviscid pulse wave speed, $\widetilde{c}_{\mathrm{i}}$, and wall viscous modulus, $\Gamma$, and the theoretical global time constant, $R_{\mathrm{T}} C_{\mathrm{T}}=1.34 \mathrm{~s}$, total compliance, $C_{\mathrm{T}}=9.60 \mathrm{~m}^{3} \mathrm{GPa}^{-1}$, and uniform outflow pressure, $P_{\text {out }}=666.5 \mathrm{~Pa}$, in the cerebral model

\begin{tabular}{|c|c|c|c|c|c|}
\hline Arterial segment & $\tilde{c}_{\mathrm{i}}\left(\mathrm{m} \mathrm{s}^{-1}\right)$ & $\Gamma(\operatorname{Pas} \mathrm{m})$ & $R_{\mathrm{T}} C_{\mathrm{T}}(\mathrm{s})$ & $C_{\mathrm{T}}\left(\mathrm{m}^{3} \mathrm{GPa}^{-1}\right)$ & $P_{\text {out }}(\mathrm{kPa})$ \\
\hline 1. Ascending aorta & $6.4(-0.6 \%)$ & $36.3(25.8 \%)$ & $1.3(-6.0 \%)$ & $8.9(-7.5 \%)$ & $0.5(-31.2 \%)$ \\
\hline 2. Thoracic aorta & $5.8(-0.6 \%)$ & $25.4(30.5 \%)$ & $1.2(-6.8 \%)$ & $8.8(-7.9 \%)$ & $0.5(-22.8 \%)$ \\
\hline 3. L. brachial & $7.0(-0.2 \%)$ & $14.1(19.1 \%)$ & $1.3(-3.8 \%)$ & $9.0(-6.1 \%)$ & $0.3(-53.8 \%)$ \\
\hline 4. R. brachial & $7.0(0.0 \%)$ & $14.2(19.3 \%)$ & $1.3(-4.4 \%)$ & $9.0(-6.4 \%)$ & $0.3(-48.0 \%)$ \\
\hline 5. L. ext. carotid & $11.5(0.0 \%)$ & $7.2(7.0 \%)$ & $1.3(0.0 \%)$ & $10.3(6.8 \%)$ & $0.1(-89.5 \%)$ \\
\hline 6. R. ext. carotid & $11.5(0.0 \%)$ & $7.2(7.0 \%)$ & $1.3(0.5 \%)$ & $10.2(6.4 \%)$ & $0.0(-95.5 \%)$ \\
\hline 7. L. PCA, P2 & $16.1(0.0 \%)$ & $4.8(4.9 \%)$ & $1.4(3.0 \%)$ & $11.2(16.2 \%)$ & $-0.1(-110.4 \%)$ \\
\hline 8. R. PCA, P2 & $16.1(0.0 \%)$ & $4.8(4.9 \%)$ & $1.4(3.0 \%)$ & $11.2(16.2 \%)$ & $-0.1(-110.4 \%)$ \\
\hline 9. L. MCA & $16.1(0.0 \%)$ & $6.7(4.7 \%)$ & $1.3(0.6 \%)$ & $10.9(13.3 \%)$ & $0.1(-91.3 \%)$ \\
\hline 10. R. MCA & $16.1(0.0 \%)$ & $6.7(4.7 \%)$ & $1.3(0.6 \%)$ & $10.8(13.0 \%)$ & $0.1(-90.9 \%)$ \\
\hline 11. L. ACA, A2 & $16.1(0.1 \%)$ & $5.6(4.8 \%)$ & $1.4(2.3 \%)$ & $11.3(17.6 \%)$ & $0.0(-103.8 \%)$ \\
\hline 12. R. ACA, A2 & $16.1(0.1 \%)$ & $5.6(4.8 \%)$ & $1.4(3.2 \%)$ & $11.3(17.9 \%)$ & $-0.1(-112.5 \%)$ \\
\hline 13. Aortic arch I & $5.9(-0.4 \%)$ & $29.2(30.7 \%)$ & $1.3(-6.3 \%)$ & $8.9(-7.7 \%)$ & $0.5(-28.1 \%)$ \\
\hline 14. Aortic arch II & $5.8(-0.3 \%)$ & $26.9(32.0 \%)$ & $1.3(-6.4 \%)$ & $8.9(-7.7 \%)$ & $0.5(-26.6 \%)$ \\
\hline 15. Brachiocephalic & $6.3(-0.2 \%)$ & $18.0(26.6 \%)$ & $1.3(-6.2 \%)$ & $8.9(-7.8 \%)$ & $0.5(-28.8 \%)$ \\
\hline 16. L. subclavian & $6.8(-0.6 \%)$ & $14.4(21.5 \%)$ & $1.3(-6.3 \%)$ & $8.9(-7.7 \%)$ & $0.5(-27.6 \%)$ \\
\hline 17. R. subclavian & $6.8(-0.4 \%)$ & $14.4(21.4 \%)$ & $1.3(-6.1 \%)$ & $8.9(-7.6 \%)$ & $0.5(-30.1 \%)$ \\
\hline 18. L. com. carotid & $8.3(-0.4 \%)$ & $12.7(13.4 \%)$ & $1.3(-5.9 \%)$ & $9.1(-5.7 \%)$ & $0.5(-32.4 \%)$ \\
\hline 19. R. com. carotid & $8.3(-0.3 \%)$ & $12.7(13.3 \%)$ & $1.3(-5.9 \%)$ & $9.0(-5.9 \%)$ & $0.4(-33.3 \%)$ \\
\hline 20. L. vertebral & $11.5(-0.3 \%)$ & $6.4(6.9 \%)$ & $1.3(-4.6 \%)$ & $9.3(-3.3 \%)$ & $0.4(-45.7 \%)$ \\
\hline 21. R. vertebral & $11.5(-0.3 \%)$ & $6.4(7.0 \%)$ & $1.3(-4.5 \%)$ & $9.3(-3.2 \%)$ & $0.4(-46.4 \%)$ \\
\hline 22. Basilar & $11.5(-0.2 \%)$ & $7.6(6.8 \%)$ & $1.3(-2.7 \%)$ & $9.9(2.6 \%)$ & $0.2(-63.9 \%)$ \\
\hline 23. L. int. carotid I & $11.5(-0.1 \%)$ & $9.5(6.9 \%)$ & $1.3(-4.1 \%)$ & $9.6(0.3 \%)$ & $0.3(-51.4 \%)$ \\
\hline 24. R. int. carotid I & $11.5(-0.1 \%)$ & $9.5(6.9 \%)$ & $1.3(-4.2 \%)$ & $9.6(-0.1 \%)$ & $0.3(-51.1 \%)$ \\
\hline 25. L. int. carotid II & $16.1(0.0 \%)$ & $9.3(4.5 \%)$ & $1.3(-2.6 \%)$ & $10.0(4.6 \%)$ & $0.2(-65.4 \%)$ \\
\hline 26. R. int. carotid II & $16.1(0.0 \%)$ & $9.3(4.5 \%)$ & $1.3(-2.7 \%)$ & $10.0(4.4 \%)$ & $0.2(-65.2 \%)$ \\
\hline 27. L. PCA, P1 & $16.1(-0.1 \%)$ & $5.0(4.2 \%)$ & $1.3(-2.2 \%)$ & $10.0(4.3 \%)$ & $0.2(-68.0 \%)$ \\
\hline 28. R. PCA, P1 & $16.1(-0.1 \%)$ & $5.0(4.2 \%)$ & $1.3(-2.3 \%)$ & $10.0(4.2 \%)$ & $0.2(-67.9 \%)$ \\
\hline 29. L. PCoA & $16.1(0.0 \%)$ & $3.3(4.6 \%)$ & $1.3(-2.8 \%)$ & $10.0(4.1 \%)$ & $0.2(-64.0 \%)$ \\
\hline 30. R. PCoA & $16.1(0.0 \%)$ & $3.3(4.6 \%)$ & $1.3(-2.8 \%)$ & $10.0(4.0 \%)$ & $0.2(-63.9 \%)$ \\
\hline 31. ACoA & $16.3(0.0 \%)$ & $3.5(5.1 \%)$ & $1.3(-1.9 \%)$ & $10.2(6.6 \%)$ & $0.2(-72.2 \%)$ \\
\hline 32. L. ACA, A1 & $16.1(0.0 \%)$ & $5.3(3.9 \%)$ & $1.3(-2.0 \%)$ & $10.2(6.0 \%)$ & $0.2(-70.4 \%)$ \\
\hline 33. R. ACA, A1 & $16.1(0.0 \%)$ & $5.3(4.0 \%)$ & $1.3(-2.0 \%)$ & $10.2(5.9 \%)$ & $0.2(-70.7 \%)$ \\
\hline
\end{tabular}

The estimates were obtained from the cardiac output and pressure and area waveforms simulated in the midpoint of each segment, using the methods described in Sects. 3.1-3.3. The corresponding theoretical values given by the parameters of the model were calculated as described in the text. The relative errors of the estimates [(estimate-theoretical)/theoretical] are shown in parentheses

$L$ left, $R$ right, ext external, int internal, com common, $P C A$ posterior cerebral artery, $M C A$ middle cerebral artery, $A C A$ anterior cerebral artery, $P C o A$ posterior communicating artery, $A C o A$ anterior communicating artery

where

$P_{\mathrm{e}}(A ; x)=P_{\mathrm{ext}}+\frac{\beta(x)}{A_{0}(x)}\left(\sqrt{A}-\sqrt{A_{0}(x)}\right)$,

$\beta(x)=\frac{4}{3} \sqrt{\pi} E(x) h(x), \quad \Gamma(x)=\frac{2}{3} \sqrt{\pi} \varphi(x) h(x)$. 
Table 2 Parameters of the TG [27] and DG [9] schemes for the single-artery and cerebral 1-D simulations

\begin{tabular}{|c|c|c|c|c|c|c|}
\hline \multirow[t]{2}{*}{ Property } & \multicolumn{2}{|c|}{ Single artery (analytical solution) } & \multicolumn{2}{|c|}{ Single artery (Gaussian inflow) } & \multicolumn{2}{|c|}{ Cerebral mode } \\
\hline & TG & DG & TG & DG & TG & DG \\
\hline Elastic time step (ms) & 10 & 10 & 0.1 & 0.1 & 0.01 & 0.05 \\
\hline Visco-elastic time step (ms) & 10 & 10 & 0.1 & 0.1 & 0.01 & 0.001 \\
\hline Grid size $(\mathrm{cm})$ & 20 & - & 0.5 & - & 0.1 & - \\
\hline Element size $(\mathrm{cm})$ & - & 100 & - & 4 & - & $2^{\mathrm{a}}$ \\
\hline Polynomial order & 1 & 3 & 1 & 4 & 1 & $3^{\mathrm{b}}$ \\
\hline Quadrature order & 2 & 3 & 2 & 4 & 2 & $3^{\mathrm{b}}$ \\
\hline
\end{tabular}

The time step is given for the purely elastic and visco-elastic models

${ }^{a}$ When physically possible

b 2 for elements smaller than $2 \mathrm{~cm}$

The elastic contribution $P_{\mathrm{e}}$ assumes a thin, homogeneous, incompressible and elastic arterial wall with thickness $h(x)$ and Young's modulus $E(x)$. Each cross section deforms axisymmetrically independently of the others (without flexural resistance) from a reference state $(P, A)=\left(P_{\text {ext }}, A_{0}\right)$, with an external pressure $P_{\text {ext }}$. Here we assume $P_{\text {ext }}=0$; i.e. $P_{\mathrm{e}}=0$ when $A=A_{0}$. The Voigt-type visco-elastic term has a wall viscosity $\varphi(x)$. The elastic pressure yields an area-dependent pulse wave speed $c$ [7],

$c=\sqrt{\frac{\beta}{2 \rho A_{0}}} A^{1 / 4}$.

Voigt-type visco-elastic models, which feature hysteresis (different stress-strain relationship for loading and unloading) and creep (continuous extension at constant load), have been extensively used [9,16,20-22]. There are, however, more complex models that also account for stress relaxation $[10,23,24]$ and the nonlinear behaviour of the wall $[25,26]$. Here we use a Voigt-type visco-elastic model since it is the simplest model to reproduce, to first approximation, the main features of visco-elastic effects on blood flow in large arteries [9,16,20-22]. It requires the estimation of only one parameter, the wall viscosity, $\varphi$, in addition to the geometry and wall stiffness of the artery.

We numerically solved Eqs. (1) and (4) in the problems studied here using a Taylor-Galerkin (TG) and a discontinuous Galerkin (DG) scheme. The former is described in [27] and the latter in [9]. Table 2 presents the properties of the TG and DG schemes for the problems studied in this work. Both schemes were verified by cross-comparison of their results in Figs. 5 and 7-11, and by comparison with analytical results given by Eqs. (15) and (16).

\subsection{Linearised 1-D formulation}

This section explores the effects on pulse waveforms of the parameters of the 1-D model. To simplify the analysis, Eqs. (1) and (4) are linearised about the reference state $\left(A, P, P_{\mathrm{e}}, Q\right)=\left(A_{0}, 0,0,0\right)$, with $\beta, A_{0}$ and $\Gamma$ constant along $x$, which yields

$$
\left\{\begin{array}{l}
C_{1 \mathrm{D}} \frac{\partial p_{\mathrm{e}}}{\partial t}+\frac{\partial q}{\partial x}=0, \\
L_{1 \mathrm{D}} \frac{\partial q}{\partial t}+\frac{\partial p_{\mathrm{e}}}{\partial x}-\gamma \frac{\partial^{2} q}{\partial x^{2}}=-R_{1 \mathrm{D}} q, \\
p=p_{\mathrm{e}}-\gamma \frac{\partial q}{\partial x}, \quad p_{\mathrm{e}}=\frac{a}{C_{1 \mathrm{D}}}, \quad \gamma=\frac{\Gamma}{A_{0}^{3 / 2}},
\end{array}\right.
$$

where $a, p, p_{\mathrm{e}}$ and $q$ are the perturbation variables for area, pressure, elastic component of pressure and flow rate, respectively; i.e. $\left(A, P, P_{\mathrm{e}}, Q\right)=\left(A_{0}+a, p, p_{\mathrm{e}}, q\right)$, and 
$C_{1 \mathrm{D}}=\frac{2 A_{0}^{3 / 2}}{\beta}, \quad L_{1 \mathrm{D}}=\frac{\rho}{A_{0}}, \quad R_{1 \mathrm{D}}=\frac{2(\zeta+2) \pi \mu}{A_{0}^{2}}$

are the elastic wall compliance, flow inertia and resistance to flow due to blood viscosity, respectively, per unit length of vessel.

Following [28, Chap. 2; 29, Chap. 6] we derive an analytical solution to (7) under the assumption of periodicity, which allows us to write $p_{\mathrm{e}}$ and $q$ as a combination of harmonic waves of the form

$p_{\mathrm{e}}(x, t)=\widehat{p}_{\mathrm{e}} e^{\mathrm{i}(\omega t-k x)}, \quad q(x, t)=\widehat{q} e^{\mathrm{i}(\omega t-k x)}$,

where $\mathrm{i}=\sqrt{-1}, k$ is the wave number, $\omega$ is the angular frequency (assumed to be real), and $\widehat{p}_{\mathrm{e}}$ and $\widehat{q}$ are the pressure and flow wave amplitudes at $(x, t)=(0,0)$, respectively.

We will focus on the solution for waves travelling in the positive $x$-direction. An identical analysis with $p_{\mathrm{e}}=$ $\widehat{p}_{\mathrm{e}} e^{\mathrm{i}(\omega t+k x)}$ and $q=\widehat{q} e^{\mathrm{i}(\omega t+k x)}$ provides the solution for waves travelling in the negative $x$-direction.

Substitution of Eq. (9) into the first two equations in (7) and combination of the resulting expressions yields

$L_{1 \mathrm{D}} \omega^{2}-\left(\gamma k^{2}+R_{1 \mathrm{D}}\right) i \omega-\frac{k^{2}}{C_{1 \mathrm{D}}}=0$,

which provides the following relation between $\omega$ and $k$ :

$\omega=\frac{\mathrm{i}\left(\gamma k^{2}+R_{1 \mathrm{D}}\right) \pm \sqrt{-\left(\gamma k^{2}+R_{1 \mathrm{D}}\right)^{2}+4 k^{2} \frac{L_{1 \mathrm{D}}}{C_{1 \mathrm{D}}}}}{2 L_{1 \mathrm{D}}}$.

The resulting phase velocity $c_{\mathrm{p}}=\omega / k$ is

$c_{\mathrm{p}}=\frac{\mathrm{i}\left(\gamma k+\frac{R_{1 \mathrm{D}}}{k}\right) \pm \sqrt{-\left(\gamma k+\frac{R_{1 \mathrm{D}}}{k}\right)^{2}+4 \frac{L_{1 \mathrm{D}}}{C_{1 \mathrm{D}}}}}{2 L_{1 \mathrm{D}}}$.

Solving Eq. (10) for $k$ yields $k^{2}=\xi e^{\mathrm{i}(\theta+2 n \pi)}$, with $n$ an integer and

$\xi=\frac{\omega C_{1 \mathrm{D}}}{1+\left(\gamma \omega C_{1 \mathrm{D}}\right)^{2}} \sqrt{\omega^{2}\left(L_{1 \mathrm{D}}-\gamma C_{1 \mathrm{D}} R_{1 \mathrm{D}}\right)^{2}+\left(R_{1 \mathrm{D}}+\gamma \omega^{2} C_{1 \mathrm{D}} L_{1 \mathrm{D}}\right)^{2}}$,

$\theta=\arctan \left(\frac{-R_{1 \mathrm{D}}-\gamma \omega^{2} C_{1 \mathrm{D}} L_{1 \mathrm{D}}}{\omega\left(L_{1 \mathrm{D}}-\gamma C_{1 \mathrm{D}} R_{1 \mathrm{D}}\right)}\right)$

Substitution of Eq. (9) into the mass conservation in (7) yields $\widehat{q}=c_{\mathrm{p}} C_{1 \mathrm{D}} \widehat{p}_{\mathrm{e}}$. Assuming that $\widehat{p}_{\mathrm{e}}$ is real, $\widehat{q}$ must be complex because $c_{\mathrm{p}}$ is complex. Therefore we can write $\widehat{q}=\operatorname{Re}(\widehat{q})+\operatorname{iIm}(\widehat{q})$, where $\operatorname{Re}$ and $\operatorname{Im}$ denote the real and imaginary parts of $\widehat{q}$, respectively. Equations (9) then become

$$
\begin{aligned}
& p_{\mathrm{e}}(x, t)=\widehat{p}_{\mathrm{e}} e^{\operatorname{Im}(k) x} e^{\mathrm{i}(\omega t-\operatorname{Re}(k) x)}, \\
& q(x, t)=(\operatorname{Re}(\widehat{q})+\mathrm{i} \operatorname{Im}(\widehat{q})) e^{\operatorname{Im}(k) x} e^{\mathrm{i}(\omega t-\operatorname{Re}(k) x)} .
\end{aligned}
$$

Their real parts represent the physical solution to (7), i.e.

$$
\begin{aligned}
& \operatorname{Re}\left(p_{\mathrm{e}}\right)=\widehat{p}_{\mathrm{e}} e^{\operatorname{Im}(k) x} \cos (\omega t-\operatorname{Re}(k) x), \\
& \operatorname{Re}(q)=e^{\operatorname{Im}(k) x}(\operatorname{Re}(\widehat{q}) \cos (\omega t-\operatorname{Re}(k) x)-\operatorname{Im}(\widehat{q}) \sin (\omega t-\operatorname{Re}(k) x)) .
\end{aligned}
$$

Equations (12-16) allow us to study the individual roles of $C_{1 \mathrm{D}}, L_{1 \mathrm{D}}, R_{1 \mathrm{D}}, \gamma$ and $\omega$ in dictating the shape of the pressure and flow pulse waves before any reflection.

Next we will focus on the local effects of the parameters of the model on the pulse waveform within an artery (Sect. 2.2), and the global effects involving all the arterial network as a whole (Sect. 2.3). 
Table 3 Typical geometrical and mechanical properties of the human aorta based on [16-18,30,32]

The calculated linear inviscid pulse wave speed is $\widetilde{c}_{\mathrm{i}}=6.17 \mathrm{~ms}^{-1}$

\begin{tabular}{ll}
\hline Property & Value \\
\hline Cross-sectional area, $A_{0}$ & $\pi \mathrm{cm}^{2}$ \\
Wall thickness, $h$ & $1.5 \mathrm{~mm}$ \\
Blood mass density, $\rho$ & $1,050 \mathrm{~kg} \mathrm{~m}^{-3}$ \\
Blood viscosity, $\mu$ & $4 \mathrm{mPa} \mathrm{s}$ \\
Velocity profile constant, $\zeta$ & 9 \\
Young's modulus, $E$ & $0.4 \mathrm{MPa}$ \\
Wall viscosity, $\varphi$ & $15 \mathrm{kPas}$ \\
\hline
\end{tabular}

\subsection{Single-artery model}

In the cardiovascular system and under normal conditions $C_{1 \mathrm{D}}>0, L_{1 \mathrm{D}}>0, R_{1 \mathrm{D}}>0$ and $\gamma>0$. Since we are interested in unsteady solutions we may also assume $\omega>0$ to obtain

$\operatorname{Im}\left(k^{2}\right)=-\frac{\omega C_{1 \mathrm{D}}}{1+\left(\gamma \omega C_{1 \mathrm{D}}\right)^{2}}\left(R_{1 \mathrm{D}}+\gamma \omega^{2} C_{1 \mathrm{D}} L_{1 \mathrm{D}}\right)<0$

from Eq. (10). Moreover we have

$\operatorname{Re}\left(k^{2}\right)=\frac{\omega^{2} C_{1 \mathrm{D}}}{1+\left(\gamma \omega C_{1 \mathrm{D}}\right)^{2}}\left(L_{1 \mathrm{D}}-\gamma C_{1 \mathrm{D}} R_{1 \mathrm{D}}\right)>0$

whenever $L_{1 \mathrm{D}}>\gamma C_{1 \mathrm{D}} R_{1 \mathrm{D}}$; i.e. fluid inertia dominates over the combined effect of wall compliance and fluid and wall viscous damping. This is equivalent to

$\rho E A_{0}>2(\zeta+2) \pi \varphi \mu$,

which is satisfied for the aortic data in Table 3; $\rho E A_{0}$ is two orders of magnitude greater than $2(\zeta+2) \pi \varphi \mu$. It is usually satisfied for arteries with smaller diameter under normal physiological conditions, since the decrease in $A_{0}$ is counterbalanced by the increase in the Young's modulus $E$ and the decrease in the velocity profile constant $\zeta$. Indeed, peripheral arteries are stiffer than the aorta [30, Chap. 7] and have a velocity profile closer to parabolic [31].

Thus, the argument in Eq. (14) is negative so that $-\pi / 2<\theta<0$. Therefore, $k=\sqrt{\xi} e^{\mathrm{i}(\theta / 2+n \pi)}$ satisfies $-\pi / 4<\theta<0, \operatorname{Re}(k)>0$ and $\operatorname{Im}(k)<0$. Equations (15) and (16) show that $\operatorname{Im}(k)$ modulates the amplitude of the pressure and flow waves; they decay exponentially with $x$ if $\operatorname{Im}(k)<0$. On the other hand, the phase shift between the flow and pressure depends on $\operatorname{Im}(\widehat{q})$.

Figure 2 shows a decrease in $\operatorname{Im}(k)$ (i.e. an increase in the pressure and flow damping with distance) with increasing frequency $\omega$, blood viscosity $\mu$ and wall viscosity $\varphi$, and decreasing Young's modulus $E$ and arterial diameter $D$. At low frequencies the damping due to $\mu$ is dominant, whereas at high frequencies the damping due to $\varphi$ dominates (Fig. 3).

Assuming blood to be inviscid $(\mu=0)$ and the arterial wall purely elastic $(\varphi=0)$, Eqs. (10) and (12) reduce to $k=\omega \sqrt{L_{1 \mathrm{D}} C_{1 \mathrm{D}}}$ and

$c_{\mathrm{p}} \equiv \widetilde{c}_{\mathrm{i}}=\frac{1}{\sqrt{L_{1 \mathrm{D}} C_{1 \mathrm{D}}}}=\sqrt{\frac{2 \sqrt{\pi} E h}{3 \rho \sqrt{A_{0}}}}$,

respectively, so that $\operatorname{Im}(k)=0, \operatorname{Im}(\widehat{q})=0$, and Eqs. (15) and (16) become $p=p_{\mathrm{e}}=\widehat{p}_{\mathrm{e}} \cos (\omega t-k x)$ and $q=\widehat{q} \cos (\omega t-k x)$. Thus, pressure and flow are in phase, have constant amplitude, and travel with frequencyindependent speed $\widetilde{c}_{\mathrm{i}}$, which is called inviscid pulse wave speed hereafter. If either $\mu>0$ or $\varphi>0, c_{\mathrm{p}}$ depends on $k$ (Eq. 12), and hence, both $\mu$ and $\varphi$ cause wave dispersion; i.e. higher-frequency waves travel faster than lower-frequency ones (Fig. 4). 

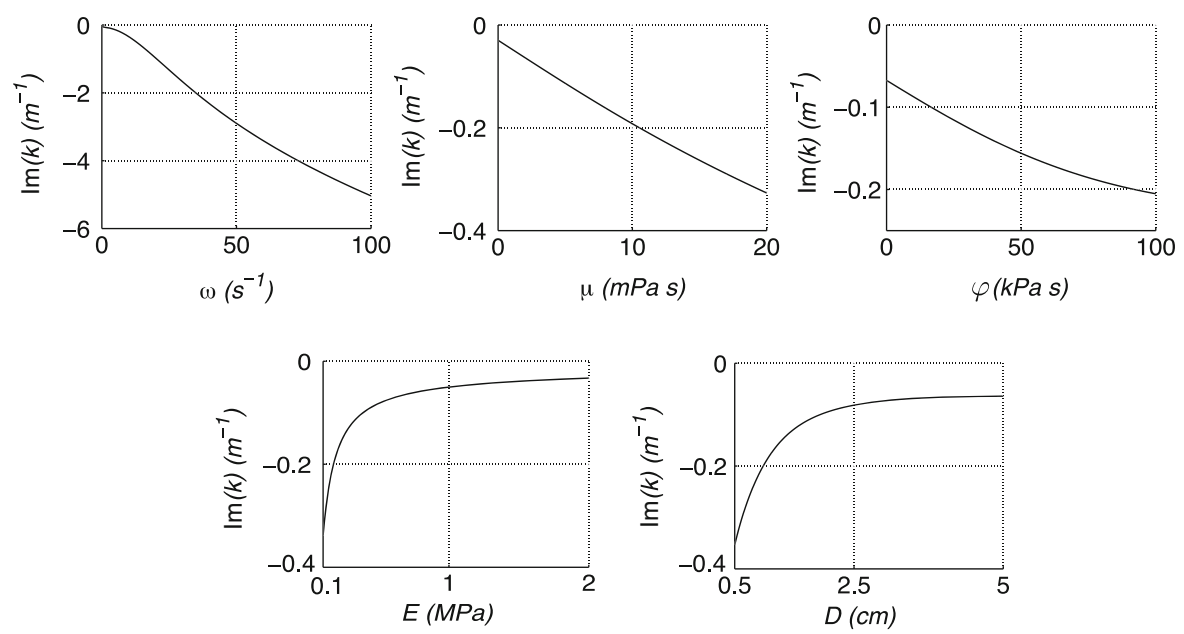

Fig. 2 Imaginary part of the wave number $\operatorname{Im}(k)$ (which dictates the pressure and flow damping with distance) calculated for the parameters given in Table 3 and a range of frequencies $\omega$ (top left), blood viscosities $\mu$ (top middle), wall viscosities $\varphi$ (top right), Young's moduli $E$ (bottom left) and arterial diameters $D$ (bottom right). In the panels with variable $\mu, \varphi, E$ or $D$, we have $\omega=\pi s^{-1}$

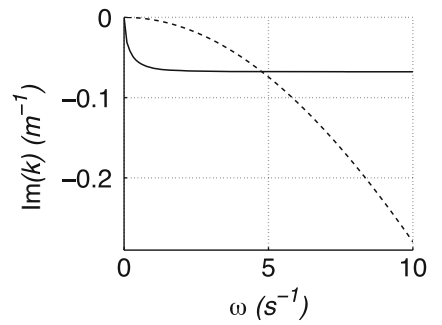

Fig. 3 Imaginary part of the wave number $\operatorname{Im}(k)$ for a range of frequencies $\omega$. It is calculated using the parameters in Table 3, except for $\varphi=0$ (solid line $)$ or $\mu=0$ (dashed line $)$
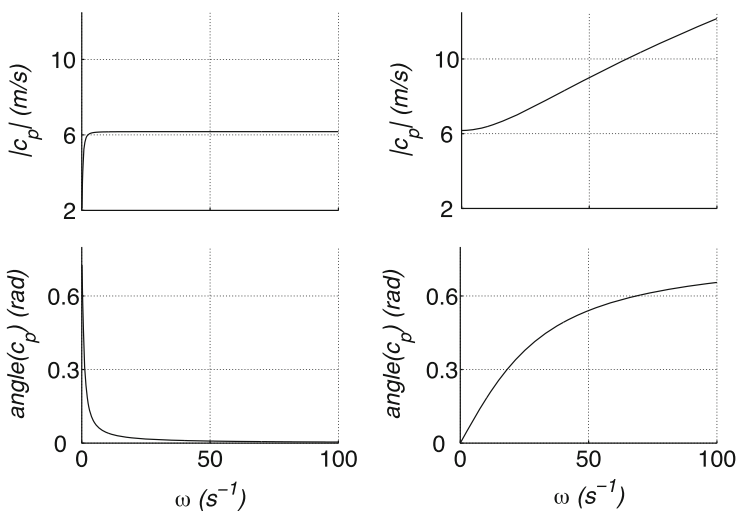

Fig. 4 Modulus (top) and phase angle (bottom) of the phase velocity $c_{\mathrm{p}}$ with the wave frequency $\omega$ in the vessel with the properties presented in Table 3 . They are obtained by solving Eq. (12) for $\omega \in(0,100]$ and $\varphi=0$ (left) or $\mu=0$ (right)

It is important to note that the phase velocity, $c_{\mathrm{p}}$, tends to the inviscid pulse wave speed, $\widetilde{c}_{\mathrm{i}}$, with increasing frequency $\omega$ (and, hence, increasing wave number $k$ ) when $\varphi=0$ and with decreasing frequency $\omega$ when $\mu=0$ [see Eqs. (12) and (18) and Fig. 4]. Moreover, the expression for $\widetilde{c}_{\mathrm{i}}$ given by Eq. (18) is also valid for non-periodic flow, as is shown in [33] using the method of characteristics.

Figure 5 shows that wall viscosity $\varphi$ (unlike blood viscosity $\mu$ ) widens the foot of a narrow Gaussian-shaped wave as it propagates in a single vessel with a completely absorbent outflow boundary condition (i.e. with a zero reflection coefficient [33]). This is because high frequencies dominate in this wave and $\mu$ causes wave dispersion at low frequencies (up to $\omega=1 \mathrm{~s}^{-1}$ for the data in Table 3; see Fig. 4, left), ${ }^{1}$ whereas $\varphi$ increases wave dispersion with increasing $\omega$ (Fig. 4, right). The results in Fig. 5 are in qualitative agreement with in vivo experiments using short trains of high frequency waves [34].

Unlike in the human pulse waveform, very high frequencies are dominant in our Gaussian-shaped wave. Due to the dependence of the pressure and flow damping, $\operatorname{Im}(k)$, on $\omega$ (see Fig. 2, left), we used a smaller wall viscosity, $\varphi=0.5 \mathrm{kPa}$, for this numerical test to be able to plot the results in the scale of Fig. 5 .

1 This is in agreement with the viscous damping due to $\mu$ being frequency independent [i.e. $\operatorname{Im}(k)$ is uniform] for $\omega>1 \mathrm{~s}^{-1}$ (Fig. 3). 


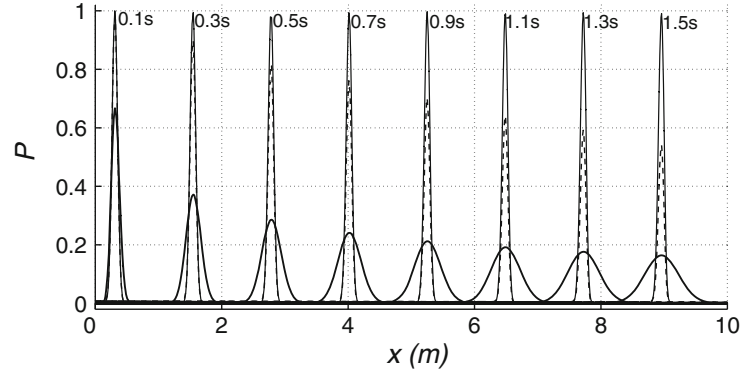

Fig. 5 Pressure with distance at the times indicated by the labels when a narrow Gaussian-shaped wave propagates from the inlet of a 10-m-long vessel with the properties presented in Table 3 (except for $\varphi=0.5 \mathrm{kPas}$ ), a volume inflow rate of $1 \mathrm{ml} \mathrm{s}^{-1}$, a completely absorbent outlet, and the initial conditions $A(x)=$ $A_{0}$ and $Q(x)=0$. Three cases are shown: elastic wall and inviscid blood (thin solid lines), visco-elastic wall and inviscid blood (thick solid lines), and elastic wall and viscous blood (dashed lines). Pressures are non-dimensionalised by the peak value of the inflow pressure

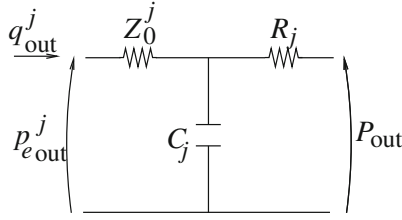

Fig. 6 The $j$-th terminal segment of the arterial network, $j=$ $2, \ldots, M$, is coupled to an RCR windkessel model relating the outgoing flow $q_{\text {out }}^{j}$ to the pressure $p_{\mathrm{e}_{\text {out }}}^{j}$. The downstream vasculature is represented by a constant peripheral resistance $R_{j}$, compliance $C_{j}$, characteristic impedance $Z_{0}^{j}$ and outflow pressure $P_{\text {out }}$

We consider this Gaussian-shaped wave to be a continuous approximation to the unit pulse $\delta\left(t-t_{0}\right), t_{0}=0.05 \mathrm{~s}$ [i.e. $\delta\left(t_{0}\right)=1$ and $\delta(t)=0$ for $t \neq t_{0}$ ]. Propagation of $\delta$ in a linear dynamic system produces the so-called impulse response function, whose convolution with any input function yields its corresponding output function directly in the time domain [35]. Thus, the pressure waveforms shown in Fig. 5 are continuous approximations to the impulse response function at different times. When nonlinear effects are small (as is the case for arterial pulse wave propagation in physiological conditions [36]), the results shown in Fig. 5 can be applied to show that blood viscosity only decreases the magnitude of the pulse waveform with distance, whereas wall viscosity also modifies its shape, especially the high-frequency components.

\subsection{Arterial network model}

We consider the arterial system to be a network of $N$ elastic and uniform arterial segments, in which pulse wave propagation is modelled using Eq. (7) and initially without assuming periodicity. To provide a synthetic description of the behaviour of each artery as a compartment of the network, we integrate the first two equations in (7) over the arterial length $l^{i}\left(x \in\left[0, l^{i}\right]\right)$ and obtain

$$
\left\{\begin{array}{l}
C_{0 \mathrm{D}}^{i} \frac{\mathrm{d} \widetilde{p}_{\mathrm{e}}^{i}}{\mathrm{~d} t}+q_{\mathrm{out}}^{i}-q_{\mathrm{in}}^{i}=0, \\
L_{0 \mathrm{D}}^{i} \frac{\mathrm{d} \widetilde{q}^{i}}{\mathrm{~d} t}+p_{\mathrm{e}_{\mathrm{out}}}^{i}-p_{\mathrm{e}}^{i}-\gamma^{i}\left(\frac{\partial q_{\mathrm{out}}^{i}}{\partial x}-\frac{\partial q_{\mathrm{in}}^{i}}{\partial x}\right)=-R_{0 \mathrm{D}}^{i} \widetilde{q}^{i},
\end{array} \quad i=1, \ldots, N,\right.
$$

where the superscript $i$ indicates the number of the segment,

$$
\begin{aligned}
& q_{\mathrm{in}}^{i}(t)=q^{i}(0, t), \quad p_{\mathrm{e}_{\mathrm{in}}}^{i}(t)=p_{\mathrm{e}}^{i}(0, t), \quad \frac{\partial q_{\mathrm{in}}^{i}}{\partial x}(t)=\frac{\partial q^{i}}{\partial x}(0, t), \\
& q_{\mathrm{out}}^{i}(t)=q^{i}\left(l^{i}, t\right), \quad p_{\mathrm{e}_{\mathrm{out}}}^{i}(t)=p_{\mathrm{e}}^{i}\left(l^{i}, t\right), \quad \frac{\partial q_{\mathrm{out}}^{i}}{\partial x}(t)=\frac{\partial q^{i}}{\partial x}\left(l^{i}, t\right),
\end{aligned}
$$

$R_{0 \mathrm{D}}^{i}=R_{1 \mathrm{D}}^{i} l^{i}, L_{0 \mathrm{D}}^{i}=L_{1 \mathrm{D}}^{i} l^{i}$ and $C_{0 \mathrm{D}}^{i}=C_{1 \mathrm{D}}^{i} l^{i}$. The space-averaged elastic pressure and flow rate over the whole segment $i$ are given by 
$\tilde{p}_{\mathrm{e}}^{i}(t)=\frac{1}{l^{i}} \int_{0}^{l^{i}} p_{\mathrm{e}} \mathrm{d} x, \quad \tilde{q}^{i}(t)=\frac{1}{l^{i}} \int_{0}^{l^{i}} q^{i} \mathrm{~d} x$.

For convenience, we assume that $i=1$ refers to the ascending aorta and $i=2$ to $M(M<N)$ refer to terminal segments. We also assume that the inlet inflow $q^{1}(0, t)$ is given and equal to the flow waveform at the inlet of the ascending aorta, $q_{\mathrm{IN}}(t)$, and that the distal ends of segments numbered from 2 to $M$ are coupled to matched RCR windkessel models relating $q_{\text {out }}^{j}$ to $p_{\mathrm{e}_{\text {out }}}^{j}, j=2, \ldots, M$, through (Fig. 6) [33]

$q_{\text {out }}^{j}\left(1+\frac{Z_{0}^{j}}{R_{j}}\right)+C_{j} Z_{0}^{j} \frac{\mathrm{d} q_{\text {out }}^{j}}{\mathrm{~d} t}=\frac{p_{\mathrm{e}_{\text {out }}}^{j}-P_{\text {out }}}{R_{j}}+C_{j} \frac{\mathrm{d} p_{\mathrm{e}_{\text {out }}}^{j}}{\mathrm{~d} t}$,

with a constant peripheral resistance $R_{j}$, compliance $C_{j}$, characteristic impedance $Z_{0}^{j}=\rho \widetilde{c}_{\mathrm{i}} / A_{0}^{j}$ and outflow pressure $P_{\text {out }}$. This is the pressure at which flow to the microcirculation ceases.

Imposing mass conservation at the junctions of the network and combining the $N$ equations of mass conservation in (19) we obtain the equation for the mass conservation for the whole network as

$q_{\mathrm{IN}}=\sum_{i=1}^{N} C_{0 \mathrm{D}}^{i} \frac{\mathrm{d} \widetilde{p}_{\mathrm{e}}^{i}}{\mathrm{~d} t}+\sum_{j=2}^{M} q_{\mathrm{out}}^{j}$.

Next we explore the role of the model parameters in the time-averaged and inertia-free solutions of Eq. (19). For this study we assume $R_{0 \mathrm{D}}^{i}=0, i=1, \ldots, N$, since it is well known that the fluid resistance in larger arteries is much smaller than peripheral resistances [30, Chap. 12].

\subsubsection{Time-averaged solution}

The time-averaged behaviour of the arterial network is not affected by (Voigt-type) wall visco-elasticity. Integrating Eq. (21) over the time interval $\left[T_{0}, T_{f}\right]$ yields

$T\left(\overline{q_{\mathrm{IN}}}-\sum_{j=2}^{M} \overline{q_{\mathrm{out}}^{j}}\right)=\sum_{i=1}^{N} C_{0 \mathrm{D}}^{i}\left[\tilde{p}_{\mathrm{e}}^{i}\left(T_{f}\right)-\tilde{p}_{\mathrm{e}}^{i}\left(T_{0}\right)\right]$,

where $\overline{(\cdot)}=\frac{1}{T} \int_{T_{0}}^{T_{f}}(\cdot) \mathrm{d} t$ is the time average over $\left[T_{0}, T_{f}\right]$, with $T=T_{f}-T_{0}$ the duration of the heartbeat, and $\overline{q_{\mathrm{IN}}}$ is the cardiac output. If the flow is periodic with a period $T$, Eqs. (20) and (22) lead to

$\overline{q_{\mathrm{IN}}}=\sum_{j=2}^{M} \overline{q_{\mathrm{out}}^{j}}=\sum_{j=2}^{M} \frac{\overline{p_{\mathrm{e}_{\mathrm{out}}^{j}}}-P_{\mathrm{out}}}{R_{j}+Z_{0}^{j}}$.

Integrating the balance of momentum in (19) over the time interval $\left[T_{0}, T_{f}\right]$ and assuming periodic flow with a period $T$ yields

$\int_{T_{0}}^{T_{f}} p_{\mathrm{e}_{\mathrm{out}}}^{i} \mathrm{~d} t-\int_{T_{0}}^{T_{f}} p_{\mathrm{e}_{\mathrm{in}}}^{i} \mathrm{~d} t=\int_{T_{0}}^{T_{f}} \gamma^{i}\left(\frac{\partial a_{\mathrm{in}}^{i}}{\partial t}-\frac{\partial a_{\mathrm{out}}^{i}}{\partial t}\right) \mathrm{d} t, \quad i=1, \ldots, N$,

where we have set $a_{\mathrm{in}}^{i}(t)=a^{i}(0, t)$ and $a_{\mathrm{out}}^{i}(t)=a^{i}\left(l^{i}, t\right)$ and used the continuity equation $\frac{\partial q^{i}}{\partial x}=-\frac{\partial a^{i}}{\partial t}$. Since the flow is periodic, $\int_{T_{0}}^{T_{f}} \frac{\partial a_{\text {in }}^{i}}{\partial t} \mathrm{~d} t$ and $\int_{T_{0}}^{T_{f}} \frac{\partial a_{\text {out }}^{i}}{\partial t} \mathrm{~d} t$ are zero, so that $\overline{p_{\mathrm{e}_{\mathrm{out}}}^{i}}=\overline{p_{\mathrm{e}}^{i}}, i=1, \ldots, N$. Moreover, since Eq. (19) holds for any length $l^{i}$, we deduce that $\overline{p_{\mathrm{e}_{\text {out }}^{i}}^{i}(t)}=\overline{p_{\mathrm{e}_{\text {in }}}^{i}(t)}=\overline{p_{\mathrm{e}}^{i}(x, t)}=\overline{p^{i}(x, t)}, i=1, \ldots, N$; i.e. the time-averaged pressure is space independent within each segment. 
Combining these results with the assumption of continuity of pressure at the junctions of the network leads to $\overline{p_{\mathrm{e}_{\text {out }}}^{j}(t)}=\overline{p^{i}(x, t)}, j=2, \ldots, M, i=1, \ldots, N$, so that Eq. (23) becomes

$\overline{p^{i}}=P_{\text {out }}+R_{\mathrm{T}} \overline{q_{\mathrm{IN}}}, \quad i=1, \ldots, N, \quad \frac{1}{R_{\mathrm{T}}}=\sum_{j=2}^{M} \frac{1}{R_{j}+Z_{0}^{j}}$,

where $R_{\mathrm{T}}$ is the net peripheral resistance of the network.

According to Eq. (22), for a given cardiac output, heart rate and peripheral properties $R_{j}, Z_{0}^{j}$ and $P_{\text {out }}, j=$ $2, \ldots, M$, wall compliances determine the time to reach a periodic state in which Eq. (24) is satisfied. Equation (24) shows that the cardiac output, outflow pressure and net resistance dictate the time-averaged pressure required to perfuse the microcirculation.

\subsubsection{Inertia-free solution}

In a linear model of the larger 55 conduit arteries in the human with inviscid flow, purely elastic walls, and RCR windkessel terminal models governed by Eq. (20), the work in [37] showed that blood pressure at any location tends to a space-independent pressure $p_{\mathrm{w}}(t)$ with increasing time in diastole. This is satisfied in general without requiring the flow to be periodic. The pressure $p_{\mathrm{w}}(t)$ is obtained by setting $L_{0 \mathrm{D}}^{i}=0, i=1, \ldots, N$, and satisfies

$q_{\mathrm{IN}}=q_{\mathrm{OUT}}+C_{\mathrm{c}} \frac{\mathrm{d} p_{\mathrm{w}}}{\mathrm{d} t}$

$q_{\text {OUT }}=C_{\mathrm{p}} \frac{\mathrm{d} p_{\mathrm{w}}}{\mathrm{d} t}+\frac{p_{\mathrm{w}}-P_{\text {out }}}{R_{\mathrm{T}}}-\sum_{j=2}^{M} \frac{C_{j} Z_{0}^{j} R_{j}}{R_{j}+Z_{0}^{j}} \frac{\mathrm{d} q_{\text {out }}^{j}}{\mathrm{~d} t}$,

$C_{\mathrm{c}}=\sum_{i=1}^{N} C_{0 \mathrm{D}}^{i}, \quad C_{\mathrm{p}}=\sum_{j=2}^{M} \frac{R_{j} C_{j}}{R_{j}+Z_{0}^{j}}$,

where $q_{\text {OUT }}(t)$ is the total outflow to the periphery driven by $p_{\mathrm{w}}, C_{\mathrm{c}}$ is the total conduit compliance, and $C_{\mathrm{p}}$ is the total peripheral compliance. The solution to Eq. (25) is

$$
p_{\mathrm{w}}=P_{\text {out }}+\left(p_{\mathrm{w}}\left(T_{0}\right)-P_{\text {out }}\right) e^{-\frac{t-T_{0}}{R_{\mathrm{T}} C_{\mathrm{T}}}}+\frac{e^{-\frac{t}{R_{\mathrm{T}} C_{\mathrm{T}}}}}{C_{\mathrm{T}}} \int_{T_{0}}^{t}\left(q_{\mathrm{IN}}\left(t^{\prime}\right)+\sum_{j=2}^{M} \frac{C_{j} Z_{0}^{j} R_{j}}{R_{j}+Z_{0}^{j}} \frac{\mathrm{d} q_{\text {out }}^{j}\left(t^{\prime}\right)}{\mathrm{d} t^{\prime}}\right) e^{\frac{t^{\prime}}{R_{\mathrm{T}} C_{\mathrm{T}}}} \mathrm{d} t^{\prime}, \quad t \geq T_{0},
$$

where $p_{\mathrm{w}}\left(T_{0}\right)$ is the pressure $p_{\mathrm{w}}$ at $t=T_{0}$ and $C_{\mathrm{T}}=C_{\mathrm{c}}+C_{\mathrm{p}}$ is the total compliance of the systemic arterial tree.

In our model, the elastic component of pressure $p_{\mathrm{e}}$ anywhere in the arterial network will tend to $p_{\mathrm{w}}(t)$ with increasing time in diastole. Since the wall viscosity term $\gamma \frac{\partial q}{\partial x}$ in Eq. (7) is linearly added to $p_{\mathrm{e}}$, the total pressure $p^{i}$ will tend to $p_{\mathrm{w}}-\gamma^{i}\left(\frac{\partial q}{\partial x}\right)^{i}, i=1, \ldots, N$, in which $\gamma^{i}$ and $\left(\frac{\partial q}{\partial x}\right)^{i}$ may be different for each arterial segment. Thus, if inertial effects are neglected, the system features a space-independent elastic pressure in diastole that depends on global quantities: the total arterial compliance, net peripheral resistance, outflow pressure, and flow rate at the inlet of the ascending aorta. ${ }^{2}$ The visco-elastic terms account for the differences in total pressure among the different arterial segments.

Figure 7 illustrates these results using the numerical solution of Eqs. (1) and (4) in the cerebral model (Fig. 1), with zero blood viscosity, so that $R_{0 \mathrm{D}}^{i}=0, i=1, \ldots, N$. The inflow boundary condition in Fig. 1 was scaled by 1,000, so that nonlinearities could be considered negligible. Pressures were then rescaled under the assumption of linearity. In diastole, the elastic pressure waveform in any artery tends to $p_{\mathrm{w}}(t)$ (Fig. 7, left), calculated using Eq. (27) with $T_{0}=0$ and $p_{\mathrm{w}}\left(T_{0}\right)=0$, and the total pressure tends to $p_{\mathrm{w}}-\gamma^{i}\left(\frac{\partial q}{\partial x}\right)^{i}, i=1, \ldots, N$ (Fig. 7, right). These results suggest that blood inertia is negligible to describe blood pressure in the last part of the cardiac cycle.

${ }^{2}$ Even though $q_{\mathrm{IN}} \approx 0$ during normal diastolic conditions, $p_{\mathrm{W}}\left(T_{0}\right)$ in Eq (27) depends on the systolic part of $q_{\mathrm{IN}}(t)$. 

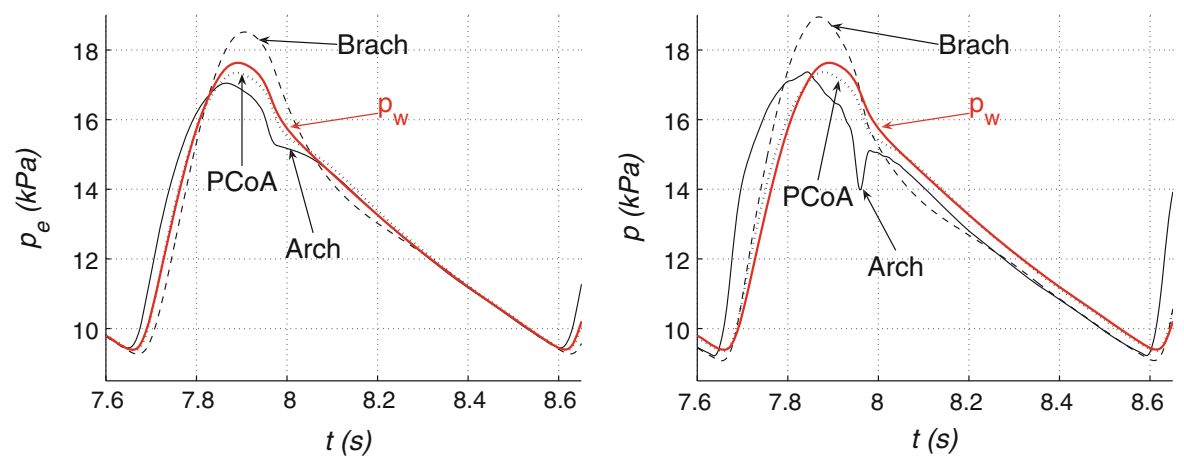

Fig. 7 Elastic (left) and total (right) pressure waveforms in the midpoint of the aortic arch I (Arch), left brachial (Brach) and left posterior communicating artery (PCoA) of the inviscid-flow, visco-elastic cerebral model (Fig. 1). Nonlinearities are neglected to compare these pressures against the space-independent pressure $p_{\mathrm{w}}$ given by Eq. (27)
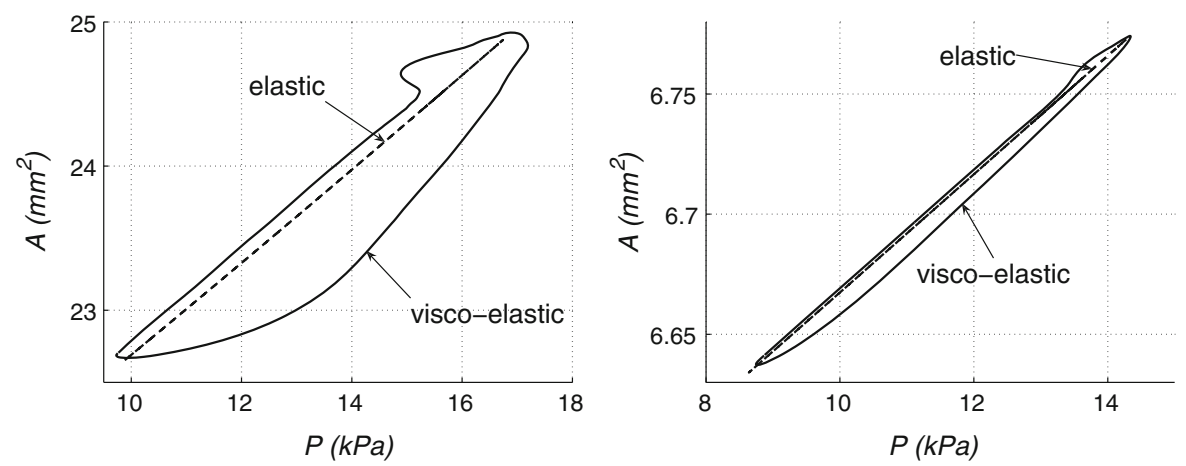

Fig. 8 Area-pressure curves in the midpoint of the right carotid artery (left) and right middle cerebral artery (right) of the viscous-fluid, nonlinear cerebral model (Fig. 1). Two cases are shown: elastic wall and visco-elastic wall with $\varphi=15 \mathrm{kPa}$. In the latter, the curves evolve in time counterclockwise

It is interesting to remark that, if $C_{j}=0, j=2, \ldots, M$ in Eq. (20) (i.e. we only have conduit compliance), Eq. (25) reduces to Frank's windkessel equation [38],

$q_{\mathrm{IN}}=\frac{p_{\mathrm{wF}_{\mathrm{F}}}-P_{\mathrm{out}}}{R_{\mathrm{T}}}+C_{\mathrm{T}} \frac{\mathrm{d} p_{\mathrm{w}_{\mathrm{F}}}}{\mathrm{d} t}$,

and $p_{\mathrm{e}}$ tends to the windkessel pressure $p_{\mathrm{w}_{\mathrm{F}}}$ with increasing time in diastole.

\section{Calculation of clinically relevant properties}

3.1 Local compliance, inviscid pulse wave speed and elastic modulus from pressure and area waveforms

According to our numerical results and for any point in our 1-D model network, $\partial A / \partial t$ tends to a constant value with increasing time in diastole. In late diastole, the elastic term in Eq. (4) leads to an approximately linear relationship between $P$ and $A$ (Fig. 8). The slope is equal to the local compliance $C_{1 \mathrm{D}}$, according to the linear tube law (7). From this slope, Eqs. (8) and (18) allow us to calculate the inviscid pulse wave speed in late diastole as

$\widetilde{c}_{\mathrm{i}}=\sqrt{\frac{A_{0}}{\rho C_{1 \mathrm{D}}}}$,

using a reasonable value for $\rho$ and approximating $A_{0}(x)$ by the time-averaged area $\bar{A}(x)$. 
If the wall thickness $h(x)$ can be measured, then the local elastic modulus $E(x)$ can be calculated as (Eq. 18)

$E=\frac{3 \bar{A}^{3 / 2}}{2 \sqrt{\pi} h C_{1 \mathrm{D}}}$.

\subsection{Local viscous modulus $\Gamma$ and wall viscosity from pressure and area waveforms}

The area-pressure curve in a viscoelastic vessel exhibits hysteresis, as shown in Fig. 8. Under the assumption of periodic flow, approximating $1 /\left(A_{0} A^{1 / 2}\right)$ by $1 / A_{\mathrm{d}}^{3 / 2}$, with $A_{\mathrm{d}}(x)$ the diastolic area, and integrating Eq. (4) over a cardiac cycle (which is indicated by $\oint$ ) leads to

$\Gamma=\frac{A_{\mathrm{d}}^{3 / 2} \oint P \mathrm{~d} A}{\oint \frac{\partial A}{\partial t} \mathrm{~d} A}$.

This equation follows from Eq. (4) since $\oint P_{\mathrm{e}} \mathrm{d} A=0$. It allows us to calculate $\Gamma(x)$ from simultaneous measurements of pressure $P$ and area $A$ at an arbitrary location in the arterial network.

If the wall thickness $h(x)$ can be measured, then the local wall viscosity $\varphi(x)$ can be calculated as (Eq. 5)

$\varphi=\frac{3 \Gamma}{2 \sqrt{\pi} h}$.

\subsection{Total compliance from the aortic root flow and a pressure waveform}

The total compliance of the systemic arterial tree $C_{\mathrm{T}}$ is a measure of the change in the total blood volume of the systemic vessels for a given change in blood pressure. From the pressure and flow waveforms at the inlet of the ascending aorta, $C_{\mathrm{T}}$ can be approximated as

$C_{\mathrm{T}}=\frac{Q_{\mathrm{p}}-Q_{0}}{P_{\mathrm{S}}-P_{\mathrm{d}}} \Delta t$

where $Q_{\mathrm{p}}$ is the peak flow rate, $Q_{0}$ is the flow rate at the onset of the systolic ejection, $P_{\mathrm{s}}$ is the systolic pressure, $P_{\mathrm{d}}$ is the diastolic pressure and $\Delta t$ is the difference between the time at $Q_{\mathrm{p}}$ and the time at $Q_{0}$. Note that $\left(Q_{\mathrm{p}}-Q_{0}\right) \Delta t$ is the blood volume pumped by the left ventricle from the onset of systole up to the time of $Q_{\mathrm{p}}$.

Alternatively, if we know the cardiac output and the pressure waveform at any other point in the systemic arterial network, we can calculate $C_{\mathrm{T}}$ using Eq. (27). During diastole, it is reasonable to assume zero flow at the inlet of the ascending aorta in normal conditions, which reduces the space-independent pressure $p_{\mathrm{w}}(t)$ to exponential functions with a time constant $R_{\mathrm{T}} C_{\mathrm{T}}$. We will explore the accuracy of estimating $R_{\mathrm{T}} C_{\mathrm{T}}$ and $P_{\text {out }}$ from an exponential fit of the form

$p_{\mathrm{w}}=P_{\text {out }}+\left(p_{\mathrm{w}}\left(T_{0}\right)-P_{\text {out }}\right) e^{-\frac{t-T_{0}}{R_{\mathrm{T}} C_{\mathrm{T}}}}, \quad t \geq T_{0}$,

to the pressure decay in diastole simulated in any arterial segment. This expression follows from Eq. (27) when all peripheral compliances $C_{j}(j=2, \ldots, M)$ are neglected. We use the MATLAB (MathWorks Inc.) function fminsearch with pressure non-dimensionalised by its maximum value and time by the period of the heartbeat, the initial conditions $R_{\mathrm{T}} C_{\mathrm{T}}=1 \mathrm{~s}$ and $P_{\text {out }}=0$, and a tolerance of $10^{-12}$.

If the cardiac output $\overline{q_{\mathrm{IN}}}$ is known, then $R_{\mathrm{T}}$ can be calculated using Eq. (24), with $\overline{p^{i}}$ calculated from the measured pressure waveform. The total compliance of the system, $C_{\mathrm{T}}$, then follows from $R_{\mathrm{T}}$ and $R_{\mathrm{T}} C_{\mathrm{T}}$.

\section{Numerical verification using nonlinear pulse waveforms}

We verify the results of the linear analysis (Sect. 4.1) and assess the accuracy of the parameter estimation techniques (Sect. 4.2) using pressure, velocity and area waveforms simulated in the cerebral network (Fig. 1) by the nonlinear 

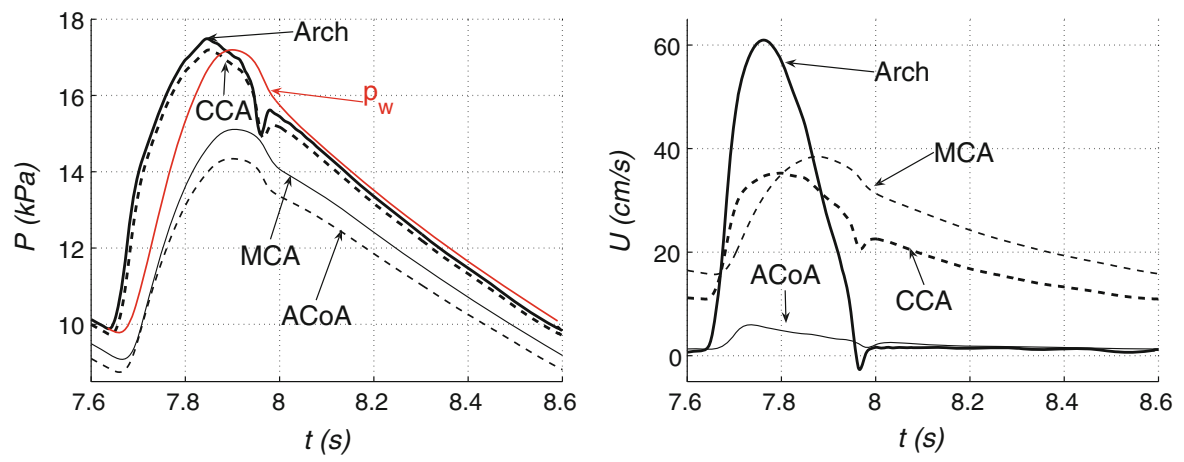

Fig. 9 Pressure (left) and velocity (right) waveforms in the midpoint of the aortic arch II (Arch), right common carotid (CCA), right middle cerebral (MCA) and anterior communicating (ACoA) arteries of the viscous-fluid, visco-elastic wall, nonlinear cerebral model (Fig. 1). The left panel shows the space-independent pressure $p_{\mathrm{w}}(t)$ given by Eq. (27) with the parameters calculated as described in the text

Eqs. (1) and (4) with a sample rate of $1 \mathrm{kHz}$. Figures 8-10 show these waveforms at several locations once the solution is periodic (after eight cardiac cycles).

\subsection{Linear analysis}

In agreement with the propagation of the narrow Gaussian-shaped waveform (Fig. 5), blood viscosity mainly affects the magnitude of pressure and velocity waveforms rather than their shapes (Fig. 10, top). At the aortic root, blood viscosity increases pressure relative to the inviscid case, due to the effect of the resistance per unit of length $R_{1 \mathrm{D}}$ (Eq. 8). This yields a net systemic resistance that is larger than the net peripheral resistance $R_{\mathrm{T}}$ by $3.5 \%$. Blood viscosity also leads to a decrease in time-averaged pressure (over the cardiac cycle) with the distance from the aortic root (Fig. 9, left). The pressure drop with distance is greater toward peripheral locations, since they have greater $R_{1 \mathrm{D}}$ due to their smaller luminal areas (Eq. 8). Moreover, $R_{1 \mathrm{D}}$ affects the distribution of the cardiac output to each terminal branch, which in turn affects the velocity waveform (Fig. 10, top right).

If blood is assumed to be inviscid, then all the arterial segments have the same time-averaged pressure $\bar{P}=$ $13.2 \mathrm{kPa}$, which depends on the cardiac output $\bar{q}_{\mathrm{IN}}$, outflow pressure $P_{\text {out }}$, and net peripheral resistance $R_{\mathrm{T}}$, in agreement with Eq. (24).

Wall viscosity smooths the pressure and flow waveforms predominantly where high frequencies are dominant (Fig. 10, bottom). Moreover, distal waveforms are smoother than proximal ones (Fig. 9), since the pulse wave is subject to a visco-elastic damping along every arterial segment, with $\operatorname{Im}(k)$ decreasing (i.e. the damping effect with distance increasing) toward the periphery.

Although wall viscosity is set to $\varphi=15 \mathrm{kPas}$ in all segments (based on data published in [16,17] for the carotid artery of normotensive men), $\Gamma /\left(A_{0} \sqrt{A}\right)$ in the tube law (4) increases toward peripheral locations. Therefore, for a given pressure $P$ and elastic contribution $P_{\mathrm{e}}, \partial A / \partial t$ will decrease as $\Gamma /\left(A_{0} \sqrt{A}\right)$ increases. This leads to smaller areas of hysteresis in the area-pressure curve in peripheral segments (Fig. 8).

The foot of the pulse waveform, which is made up of high frequencies, arrives at the measuring site earlier in the visco-elastic model (Fig. 10, bottom). This is in agreement with the increase in wave dispersion with frequency shown in Fig. 4 (right). In early systole, the expansion of the vessel wall $(\partial A / \partial t>0)$ leads to a larger pressure in the visco-elastic model than in the purely elastic one (Fig. 10, bottom left), which is in agreement with Eq. (4). The opposite effect is observed when the vessel wall relaxes $(\partial A / \partial t<0)$ in late systole and diastole.

Figure 9 (left) shows the tendency in time of all pressure waveforms to a similar exponential shape (except for the magnitude due to viscous blood dissipation) in diastole. This shape is well captured by a space-independent $p_{\mathrm{w}}(t)$ calculated using Eq. (27) with an asymptote $P_{\text {out }}=666.5 \mathrm{~Pa}, p_{\mathrm{w}}\left(T_{0}\right)$ taken to be equal to the 

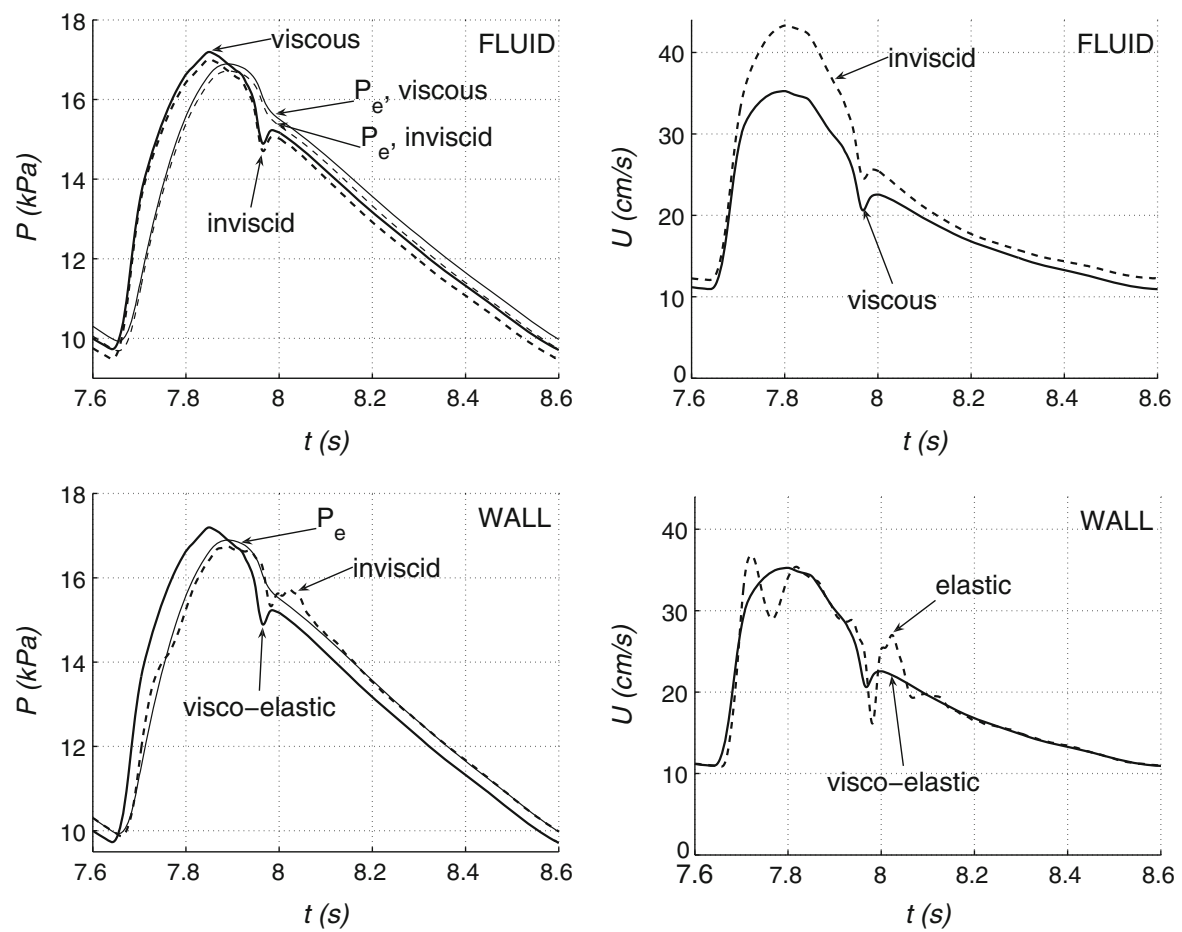

Fig. 10 Pressure (left) and velocity (right) waveforms in the midpoint of the right common carotid artery of the nonlinear cerebral model (Fig. 1), comparing the models with viscous and inviscid fluid (top) and elastic wall and visco-elastic wall (bottom). $P_{\mathrm{e}}$ is the elastic contribution to pressure

pressure at the aortic root at the beginning of the cardiac cycle $\left(T_{0}=7.63 \mathrm{~s}\right), R_{\mathrm{T}}=139.11 \mathrm{MPa} \mathrm{s} \mathrm{m}^{-3}$ calculated using $R_{\mathrm{T}}=\left(\overline{p^{i}}-P_{\mathrm{out}}\right) / \overline{q_{\mathrm{IN}}}$ from Eq. (24), with the time-averaged pressure at the aortic root, $C_{\mathrm{p}}$ calculated using Eq. (26), and $C_{\mathrm{c}}=\sum_{i=1}^{N} C_{1 \mathrm{D}}^{i} l^{i}=5.15 \mathrm{~m}^{3} \mathrm{GPa}^{-1}$, with $C_{1 \mathrm{D}}^{i}=2 A_{0}^{i}\left(\bar{A}^{i}\right)^{1 / 2} / \beta^{i}$ and $\bar{A}^{i}$ the mean area over one cardiac cycle $(i=1, \ldots, N)$. The resulting time constant and total compliance are $R_{\mathrm{T}} C_{\mathrm{T}}=1.34 \mathrm{~s}$ and $C_{\mathrm{T}}=9.60 \mathrm{~m}^{3} \mathrm{GPa}^{-1}$, respectively. This result is in agreement with observations that human [39] and canine [40] in vivo pressure waveforms are remarkably uniform in about the last two-thirds of diastole in normal conditions.

\subsection{Estimation techniques}

Table 1 shows the estimates of the inviscid pulse wave speed, $\widetilde{c}_{\mathrm{i}}$, wall viscous modulus, $\Gamma$, time constant, $R_{\mathrm{T}} C_{\mathrm{T}}$, total compliance, $C_{\mathrm{T}}$, and outflow pressure, $P_{\text {out }}$. They were obtained from the cardiac output and pressure and area waveforms simulated in the midpoint of each segment, using the methods described in Sects. 3.1-3.3 [fitting Eq. (34) to calculate $C_{\mathrm{T}}$ ]. The estimate of $C_{\mathrm{T}}$ using Eq. (33) is $C_{\mathrm{T}}=9.00 \mathrm{~m}^{3} \mathrm{GPa}^{-1}$. These estimations are compared with the corresponding theoretical values obtained from the parameters of the model using Eq. (6) for $\widetilde{c}_{\mathrm{i}}$ (with $A$ equal to its time-averaged value over one cardiac cycle, $\bar{A}$ ), Eq. (5) for $\Gamma$, the calculations described in the paragraph above for $R_{\mathrm{T}} C_{\mathrm{T}}=1.34 \mathrm{~s}$ and $C_{\mathrm{T}}=9.60 \mathrm{~m}^{3} \mathrm{GPa}^{-1}$, and the prescribed $P_{\text {out }}=$ $666.5 \mathrm{~Pa}$.

In any segment, $\widetilde{c}_{\mathrm{i}}$ is estimated with an error smaller than $1 \%$. The estimates of $\widetilde{c}_{\mathrm{i}}$ are not very sensitive to errors in the blood density $\rho$; if a $\pm 5 \%$ error is introduced in $\rho$, errors in the estimates are smaller than $3 \%$. $\Gamma$ is overestimated, especially in proximal vessels, since they undergo greater changes in cross-sectional area than distal ones, thus increasing the approximation error in Eq. (31). 

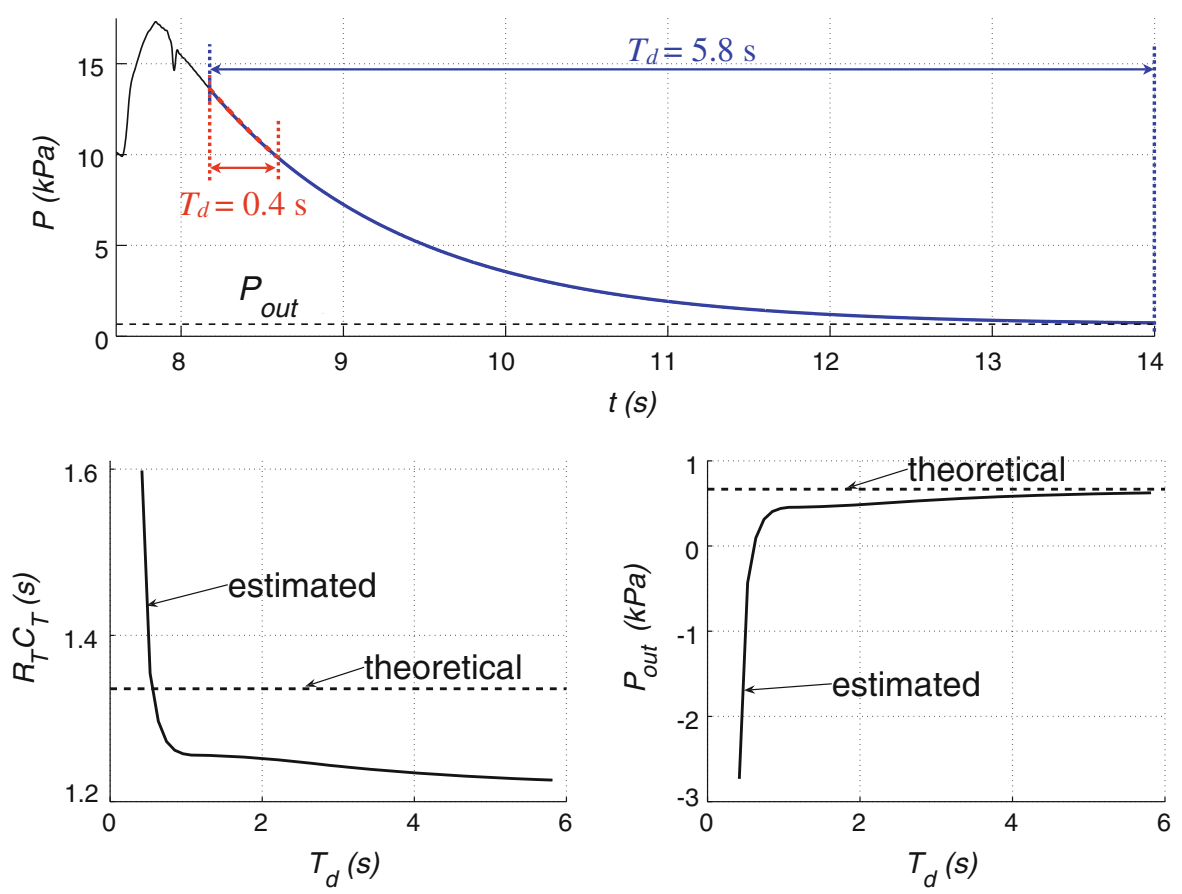

Fig. 11 Top Pressure waveform at the aortic root of the viscous-fluid, visco-elastic wall, nonlinear cerebral model (Fig. 1). A zero volume flow rate was prescribed after $t=8.6 \mathrm{~s}$. Bottom Estimates of $R_{\mathrm{T}} C_{\mathrm{T}}$ (left) and $P_{\mathrm{out}}$ (right) with the diastolic period $T_{\mathrm{d}}$ : from the last two-thirds of diastole $\left(T_{\mathrm{d}}=0.4 \mathrm{~s}\right)$ to $T_{\mathrm{d}}=5.8 \mathrm{~s}$ (see upper panel). The corresponding theoretical values are shown in dashed lines

The accuracy of the estimates of $R_{\mathrm{T}} C_{\mathrm{T}}$ and $P_{\text {out }}$ depends upon the diastolic region used for the exponential fitting. Figure 11 (top) shows the simulated pressure decay at the aortic root when the cardiac ejection is stopped $\left(q_{\mathrm{IN}}=0\right)$ after $t=8.6 \mathrm{~s}$. The lower panels show the sensitivity of the estimates of $R_{\mathrm{T}} C_{\mathrm{T}}$ and $P_{\mathrm{out}}$ to changes in the diastolic period $T_{\mathrm{d}}$. As $T_{\mathrm{d}}$ increases, the estimates of $P_{\text {out }}$ approach the theoretical values. The estimates of $R_{\mathrm{T}} C_{\mathrm{T}}$ improve up to the threshold $\widetilde{T_{\mathrm{d}}}=0.6 \mathrm{~s}$. They worsen for $T_{\mathrm{d}}>\widetilde{T}_{\mathrm{d}}$ since in our nonlinear model $C_{\mathrm{T}}$ decreases as the pressure drops, in agreement with the in vivo system [41], and $R_{\mathrm{T}}$ decreases as the wall friction $f$ drops with the decreasing blood velocity. Different thresholds $\widetilde{T}_{\mathrm{d}}$ are obtained at other locations (e.g. in the midpoint of the thoracic, carotid and middle cerebral arteries $\widetilde{T}_{\mathrm{d}}$ is equal to $0.5,0.6$ and $1.7 \mathrm{~s}$, respectively). In Table 1 we used $T_{\mathrm{d}}=1.5 \mathrm{~s}$, which is a feasible value in vivo, e.g. when a missing or ectopic beat occurs (as observed even in healthy subjects) [42] or when drugs are administered to temporally stop the heartbeat in animal experiments.

In agreement with Fig. 11 (bottom, right), $P_{\text {out }}$ is underestimated in all the segments, especially in distal vessels. The error in the estimates of $R_{\mathrm{T}} C_{\mathrm{T}}$ is smaller than $7 \%$ in any segment; it is overestimated in all cerebral vessels and underestimated in more proximal vessels. Despite the large errors in the estimates of $P_{\text {out }}$, $C_{\mathrm{T}}$ is estimated with a relative error smaller than $8 \%$ in all segments, except for the cerebral arteries. Relative errors for $C_{\mathrm{T}}$ are smaller than $13 \%$ in non-cerebral arteries if a $\pm 5 \%$ error is introduced in the cardiac output, $\overline{q_{\mathrm{IN}}}$.

The high symmetry of the system yields similar estimates in those arteries that are present on the right and left circulations.

\section{Concluding remarks}

We have used the 1-D formulation to investigate mechanisms underlying the effect on pressure, flow and area waveforms of several physical properties of the cardiovascular system. Based on these investigations, we have derived methods to calculate the local visco-elastic properties of the arterial wall, and the total arterial compliance, net resistance, time constant and peripheral outflow pressure of the systemic arterial tree from data that can be 
measured in vivo. Knowledge of these properties is clinically important for the diagnosis and treatment of disease and for providing patient-specific parameters to computational models, which have the potential to support the prognosis and design of therapeutic interventions.

We have shown that the damping effect on the pulse waveform increases with increasing frequency, blood viscosity and wall viscosity, and decreasing Young's modulus and luminal area. Blood viscosity causes wave dispersion for the lower frequencies of pulse waveforms, decreasing the pulse wave magnitude, whereas wall viscosity, $\varphi$, increases wave dispersion with increasing frequency, smoothing pulse waveforms predominantly where higher frequencies are dominant. Although all the arteries studied here have the same $\varphi$, distal pulse waveforms are smoother and have smaller areas of hysteresis in the area-pressure curve than proximal ones. This is due to the combined effect of the visco-elastic damping acting along every arterial segment, and $\Gamma(x) / A_{0}(x) \sqrt{A}$ increasing as the cross-sectional area of the lumen decreases. Moreover, $\varphi$ amplifies the pulse pressure due to the expansion of the vessel wall during early systole.

We have also shown that the inertial effects of blood flow become negligible during approximately the last twothirds of diastole, when pressures tend to the space-independent shape $p_{\mathrm{w}}(t)$ dictated by global quantities (cardiac ejection, total compliance, net peripheral resistance, and outflow pressure) and the local viscous modulus. Although $p_{\mathrm{w}}(t)$ fails to reproduce the wave-like nature of pulse propagation during systole and early diastole, it provides a zero-order approximation to the nonlinear pressure $P(x, t)$ in larger systemic arteries. This approximation allows us to study the effect on the pressure waveform of the cardiac ejection, total compliance, net peripheral resistance, and outflow pressure.

We have focussed the exposition on understanding mechanisms underlying arterial pulse wave propagation in the upper body, but similar mechanisms are expected in most of the conduit arterial network, since the mathematical problem is conceptually the same. The coronary arteries are an exception due to the effect of myocardial contraction.

We have described methods to calculate the local compliance, inviscid pulse wave speed and wall visco-elastic moduli from simultaneous pressure and area waveforms, and the total compliance, net resistance and outflow pressure from the flow at the aortic root and a pressure waveform at an arbitrary arterial location. These methods rely on the ability of the 1-D formulation to capture the main features of pulse waveforms in large arteries, which is supported by several studies $[9,23,25,36,43]$, and the feasibility of taking accurate measurements of the in vivo data required for the calculations.

In vivo measurements are currently available for only some human arteries. The flow at the aortic root can be measured using Doppler ultrasound [44, p. 38] and magnetic resonance (MR) [45]. Blood pressure can be recorded noninvasively in superficial arteries, such as carotid, brachial, radial and femoral, using applanation tonometry $[16,25,26,46]$. Invasive measurements can be obtained in the aorta and other extracranial arteries using pressuresensing catheters [14]. The internal luminal area can be measured in superficial arteries using ultrasound-based echo-tracking [16,26,47] and MR [45]. Invasive measurements using piezoelectric crystal transducers have been carried out in animal experiments [26].

According to the results in Table 1, accurate measurements of pressure and area at the common carotid artery and the cardiac output could lead to errors smaller than $6 \%$ in the estimates of the local compliance, total systemic compliance, and time constant at the aortic root.

For patient-specific 1-D simulations we also need arterial lengths and mean cross-sectional areas, which can be obtained from medical images, such as MR imaging [10,48,49]. The wall thickness is necessary to calculate the local Young's modulus, Eq. (30), and wall viscosity, Eq. (32). It is not necessary, however, to calculate local pulse wave speeds (or compliances $C_{1 \mathrm{D}}$ ) and viscous moduli $\Gamma$, which are sufficient to simulate the propagation of the pressure and flow waveforms using the 1-D formulation.

Given the amount of in vivo data usually available in the clinic, not all the parameters of the 1-D model can be calculated using the methods proposed here. Optimisation techniques as those proposed in [10,11] can be used to calculate the rest of parameters. We believe, however, that mechanical-based algorithms, as those described here, should first be used to determine the total compliance and net resistance of the system, outflow pressures, and local compliances and viscous moduli at as many locations as possible. For example, knowledge of the wave speed, $\widetilde{c}_{\mathrm{i}}$, and luminal area at a few locations allows us to relate them through a least-squares fitting $[25,31,50]$, so that $\widetilde{c}_{\mathrm{i}}$ 
can be inferred elsewhere in the system given local areas from medical images. Once the net peripheral resistance and compliance are known, we can distribute them among the terminal branches based on mechanical principles $[8,33]$.

Analysis of in vivo data is beyond the scope of this study. We have used numerical waveforms to verify our results, which are free of measurement and alignment errors. Moreover, the numerical model provides theoretical values to compare with the estimates. However, numerical waveforms are an approximation to in vivo waveforms. This is, therefore, a limitation of our study, and a full verification of our results using in vivo data remains to be done in future work. Future work could also carry out a similar analysis using more complex visco-elastic models of the arterial wall that account for stress relaxation $[10,23,24]$ and the nonlinear behaviour of the arterial wall $[25,26]$.

This work has proposed methods to calculate physical quantities of the system that are important for assessing cardiovascular function and running patient-specific simulations of pulse wave propagation in the systemic arterial network.

Acknowledgments This work was supported by a British-Italian partnership programme for young researchers (British Council/ MIUR). Jordi Alastruey was also funded by a British Heart Foundation Intermediate Basic Science Research Fellowship (FS/09/030/27812) and the Centre of Excellence in Medical Engineering funded by the Wellcome Trust and EPSRC under grant number WT 088641/Z/09/Z. Luca Formaggia gratefully acknowledges the support of MIUR through a PRIN07 grant.

Open Access This article is distributed under the terms of the Creative Commons Attribution License which permits any use, distribution, and reproduction in any medium, provided the original author(s) and the source are credited.

\section{References}

1. Blacher J, Asmar R, Djane S, London GM, Safar ME (1999) Aortic pulse wave velocity as a marker of cardiovascular risk in hypertensive patients. Hypertension 33:1111-1117

2. Laurent S, Boutouyrie P, Asmar R, Gautier I, Laloux B, Guize L, Ducimetiere P, Benetos A (2001) Aortic stiffness is an independent predictor of all-cause and cardiovascular mortality in hypertensive patients. Hypertension 37:1236-1241

3. Meaume S, Rudnichi A, Lynch A, Bussy C, Sebban C, Benetos A, Safar ME (2001) Aortic pulse wave velocity as a marker of cardiovascular disease in subjects over 70 years old. J Hypertens 19:871-877

4. Randall SO, Esler MD, Calfee RV, Bulloch GF, Maisel AS, Culp B (1976) Arterial compliance in hypertension. Aust N Z J Med 6:49-59

5. Simon AC, Safar ME, Levenson JA, London GM, Levy BI, Chau NP (1979) An evaluation of large arteries compliance in man. Am J Physiol 237:H550-H554

6. Armentano RL, Barra JG, Pessana FM, Craiem DO, Graf S, Santana DB, Sanchez RA (2007) Smart smooth muscle spring-dampers. Smooth muscle smart filtering helps to more efficiently protect the arterial wall. IEEE Eng Med Biol Mag 26:62-70

7. Peiró J, Veneziani A (2009) Reduced models of the cardiovascular system. In: Formaggia L, Quarteroni A, Veneziani A (eds) Cardiovascular mathematics. Modeling and simulation of the circulatory system. Springer, Milano pp 347-394

8. van de Vosse FN, Stergiopulos N (2011) Pulse wave propagation in the arterial tree. Annu Rev Fluid Mech 43:467-499

9. Alastruey J, Khir AW, Matthys KS, Segers P, Sherwin SJ, Verdonck P, Parker KH, Peiró J (2011) Pulse wave propagation in a model human arterial network: assessment of 1-D visco-elastic simulations against in vitro measurements. J Biomech 44:2250-2258

10. Devault K, Gremaud P, Novak V, Olufsen M, Vernières G, Zhao P (2008) Blood flow in the circle of Willis: modeling and calibration. Multiscale Model Simul 7:888-909

11. Martin V, Clément F, Decoene A, Gerbeau JF (2005) Parameter identification for a one-dimensional blood flow model. ESAIM Proc 14:174-200

12. Leguy CAD, Bosboom EMH, Gelderblom H, Hoeks APG, van de Vosse FN (2010) Estimation of distributed arterial mechanical properties using a wave propagation model in a reverse way. Med Eng Phys 32:957-967

13. Khir AW, O’Brien A, Gibbs JSR, Parker KH (2001) Determination of wave speed and wave separation in the arteries. J Biomech 34:1145-1155

14. Davies JE, Whinnett ZI, Francis DP, Willson K, Foale RA, Malik IS, Hughes AD, Parker KH, Mayet J (2006) Use of simultaneous pressure and velocity measurements to estimate arterial wave speed at a single site in humans. Am J Physiol Heart Circ Physiol 290:H878-H885

15. Alastruey J, Parker KH, Peiró J, Byrd SM, Sherwin SJ (2007) Modelling the circle of Willis to assess the effects of anatomical variations and occlusions on cerebral flows. J Biomech 40:1794-1805

16. Armentano R, Megnien JL, Simon A, Bellenfant F, Barra J, Levenson J (1995) Effects of hypertension on viscoelasticity of carotid and femoral arteries in humans. Hypertension 26:48-54 
17. Gariepy J, Massonneau M, Levenson J, Heudes D, Simon A (1993) Evidence for in vivo carotid and femoral wall thickening in human hypertension. Groupe de Prévention Cardio-vasculaire en Médecine du Travail. Hypertension 22:111-118

18. Smith NP, Pullan AJ, Hunter PJ (2001) An anatomically based model of transient coronary blood flow in the heart. SIAM J Appl Math 62:990-1018

19. Brook BS, Falle SAEG, Pedley TJ (1999) Numerical solutions for unsteady gravity-driven flows in collapsible tubes: evolution and roll-wave instability of a steady state. J Fluid Mech 396:223-256

20. Armentano R, Barra J, Levenson J, Simon A, Pichel RH (1995) Arterial wall mechanics in conscious dogs: assessment of viscous, inertial, and elastic moduli to characterize aortic wall behavior. Circ Res 76:468-478

21. Craiem D, Graf S, Pessana F, Grignola J, Bia D, Gines F, Armentano R (2005) Cardiovascular engineering: modelization of ventricular arterial interaction in systemic and pulmonary circulation. Lat Am Appl Res 35:111-114

22. Čanić S, Tambača J, Guidoboni G, Mikelić A, Hartley C, Rosenstrauch D (2006) Modeling viscoelastic behavior of arterial walls and their interaction with pulsatile blood flow. SIAM J Appl Math 67:164-193

23. Bessems D, Giannopapa CG, Rutten MCM, van de Vosse FN (2008) Experimental validation of a time-domain-based wave propagation model of blood flow in viscoelastic vessels. J Biomech 41:284-291

24. Valdez-Jasso D, Haider MA, Banks HT, Santana DB, German YZ, Armentano RL, Olufsen MS (2009) Analysis of viscoelastic wall properties in ovine arteries. IEEE Trans Biomed Eng 56:210-219

25. Reymond P, Merenda F, Perren F, Rüfenacht D, Stergiopulos N (2009) Validation of a one-dimensional model of the systemic arterial tree. Am J Physiol Heart Circ Physiol 297:H208-H222

26. Valdez-Jasso D, Bia D, Zócalo Y, Armentano RL, Haider MA, Olufsen MS (2011) Linear and nonlinear viscoelastic modeling of aorta and carotid pressure-area dynamics under in vivo and ex vivo conditions. Ann Biomed Eng 39:1438-1456

27. Formaggia L, Lamponi D, Quarteroni A (2003) One-dimensional models for blood flow in arteries. J Eng Math 47:251-276

28. Passerini T (2009) Computational hemodynamics of the cerebral circulation: multiscale modeling from the circle of Willis to cerebral aneurysms. Ph.D. thesis, Politecnico di Milano, Italy

29. Giannopapa CG (2004) Fluid-structure interaction in flexible vessels. Ph.D. thesis, University of London, UK

30. Caro CG, Pedley TJ, Schroter RC, Seed WA (2011) The mechanics of the circulation, 2nd edn. Cambridge University Press, Cambridge

31. Olufsen MS (1999) Structured tree outflow condition for blood flow in larger systemic arteries. Am J Physiol 276:H257-H268

32. Westerhof N, Bosman F, de Vries CJ, Noordergraaf A (1969) Analog studies of the human systemic arterial tree. J Biomech 2:121-143

33. Alastruey J, Parker KH, Peiró J, Sherwin SJ (2008) Lumped parameter outflow models for 1-D blood flow simulations: effect on pulse waves and parameter estimation. Commun Comput Phys 4:317-336

34. Anliker M, Histand MB, Ogden E (1968) Dispersion and attenuation of small artificial pressure waves in the canine aorta. Circ Res 23:539-551

35. Lynn PA, Fuerst W (1989) Introductory digital signal processing with computer applications. Wiley, New York

36. Matthys KS, Alastruey J, Peiró J, Khir AW, Segers P, Verdonck PR, Parker KH, Sherwin SJ (2007) Pulse wave propagation in a model human arterial network: assessment of 1-D numerical simulations against in vitro measurements. J Biomech 40:3476-3486

37. Alastruey J (2010) On the mechanics underlying the reservoir-excess separation in systemic arteries and their implications for pulse wave analysis. Cardiovasc Eng 10:176-189

38. Frank O (1899) Die Grundform des arteriellen Pulses. Erste AbhandlungMathematische Analyse Z Biol 37:483-526

39. Aguado-Sierra J, Alastruey J, Wang J-J, Hadjiloizou N, Davies JE, Parker KH (2008) Separation of the reservoir and wave pressure and velocity from measurements at an arbitrary location in arteries. Proc Inst Mech Eng H 222:403-416

40. Wang J-J, O’Brien AB, Shrive NG, Parker KH, Tyberg JV (2003) Time-domain representation of ventricular-arterial coupling as a windkessel and wave system. Am J Physiol Heart Circ Physiol 284:H1358-H1368

41. Roy C (1880) The elastic properties of the arterial wall. J Physiol (Lond) 3:125-159

42. Parker KH (2009) An introduction to wave intensity analysis. Med Biol Eng Comput 47:175-188

43. Olufsen MS, Peskin CS, Kim WY, Pedersen EM, Nadim A, Larsen J (2000) Numerical simulation and experimental validation of blood flow in arteries with structured-tree outflow conditions. Ann Biomed Eng 28:1281-1299

44. Oates C (2001) Cardiovascular haemodynamics and Doppler waveforms explained. Greenwich Medical Media, London

45. Ibrahim E-SH, Johnson KR, Miller AB, Shaffer JM, White RD (2010) Measuring aortic pulse wave velocity using high-field cardiovascular magnetic resonance: comparison of techniques. J Cardiovasc Magn Reson 12:26-39

46. Zambanini A, Cunningham SL, Parker KH, Khir AW, Thom SA, Hughes AD (2005) Wave-energy patterns in carotid, brachial, and radial arteries: a noninvasive approach using wave-intensity analysis. Am J Physiol Heart Circ Physiol 289:H270-H276

47. Levenson JA, Peronneau PP, Simon AC, Safar ME (1981) Pulsed Doppler: determination of diameter, blood flow velocity and volumic flow of brachial artery in man. Cardiovasc Res 15:164-170

48. Cebral JR, Castro MA, Soto O, Löhner R, Alperin N (2003) Blood-flow models of the circle of Willis from magnetic resonance data. J Eng Math 47:369-386

49. Moore SM, David T, Chase JG, Arnold J, Fink J (2006) 3D models of blood flow in the cerebral vasculature. J Biomech 39:14541463

50. Alastruey J, Nagel SR, Nier B, Hunt AAE, Weinberg PD, Peiró J (2009) Modelling pulse wave propagation in the rabbit systemic circulation to assess the effects of altered nitric oxide synthesis. J Biomech 42:2116-2123 